\title{
A Biogically Plausible Neuron Model of Mental Rotation
}

by

Sean N. Riley

A thesis submitted to the Faculty of Graduate and Postdoctoral Affairs in partial fulfillment of the requirements for the degree of

Master

of

Cognitive Science

Carleton University

Ottawa, Ontario

(C) 2018, Sean N. Riley 


\begin{abstract}
This thesis presents a biologically plausible account of mental rotation. To this end, there is evidence that mental rotation is a spatial imagery task that can invoke a variety of strategies, depending on the nature of the stimuli. This thesis uses simple but unfamiliar stimuli, which engenders a continuous, whole-unit rotation. The model is comprised of 43,000 simulated neurons spread across a variety of neuron ensembles. These ensembles work together to form a neuronal representation of the spatial map entailed by the stimuli, then rotates that spatial map into a series of new orientations according to simulated movement along an intended axis of rotation. Two sets of simulations were run: one focusing on the biological accuracy of spatial maps, with the second focusing on the biological accuracy of neurons. Overall, both sets of simulations were able to re-produce the reaction times found in behavioural studies of mental rotation.
\end{abstract}




\section{Acknowledgements}

This thesis would not have been possible without the guidance of Dr. Jim Davies and Dr. Robert West, both of whom provided invaluable insights into the model and the theory that underpins it. Moreover, without the support of my family I would likely be shouting at baby ducks along the banks of the Rideau River, which would probably anger Ducks Unlimited... I don't want to anger Ducks Unlimited. Finally, a big shout-out to Spotify for providing countless hours of background noise whilst I sat in my beloved desk chair working on this project (aka, time spent not shouting at baby ducks).

Thank-you all for everything. 


\section{Table of Contents}

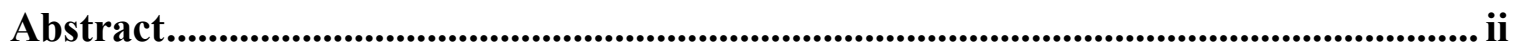

Acknowledgements ............................................................................................................................. iii

Table of Contents ........................................................................................................ iv

List of Tables ................................................................................................................................ vi

List of Illustrations..................................................................................................... vii

1 Chapter: Introduction ....................................................................................................... 8

2 Chapter: Mental Imagery .............................................................................................. 9

$2.1 \quad$ Depictive Framework ..................................................................................

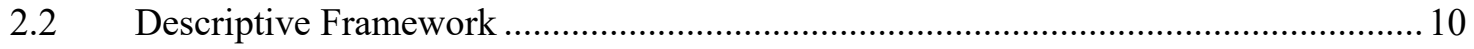

2.3 Behavioural Findings.......................................................................................... 12

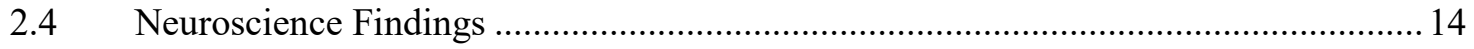

2.4.1 Topological Organization of the Primary Visual Cortex …………………………... 14

2.4.2 Shared Correlates of Visual Perception and Mental Imagery ………........................15

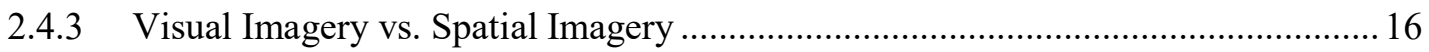

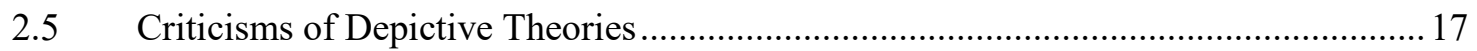

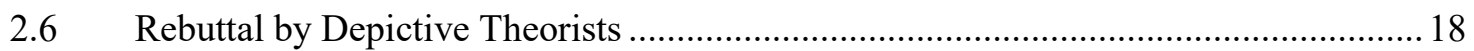

2.7 Kosslyn's Theory of Mental Imagery ..................................................................2 20

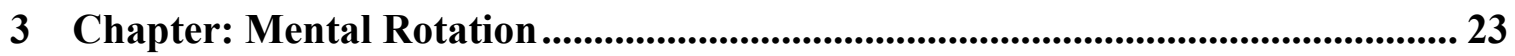

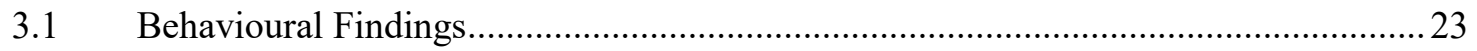

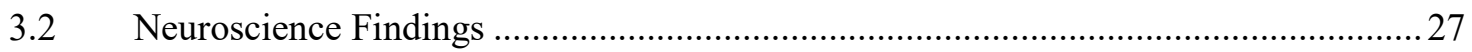

4 Chapter: Model Representation ................................................................................... 31

5 Chapter: The Neural Engineering Framework ......................................................... 35

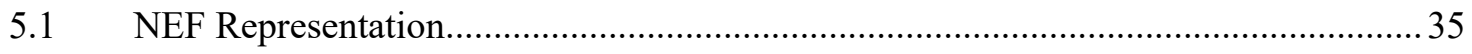




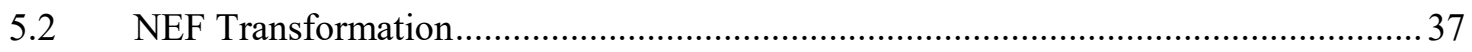

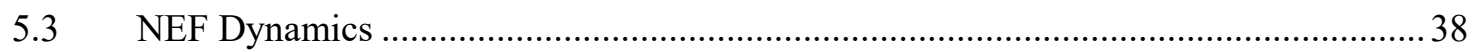

6 Chapter: Overview of Model .......................................................................... 40

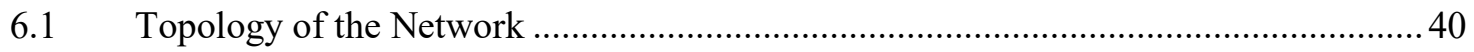

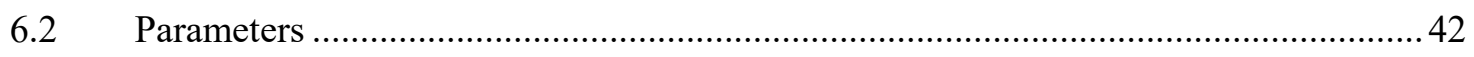

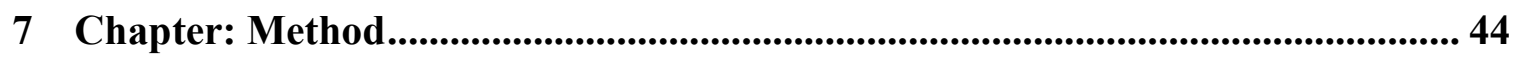

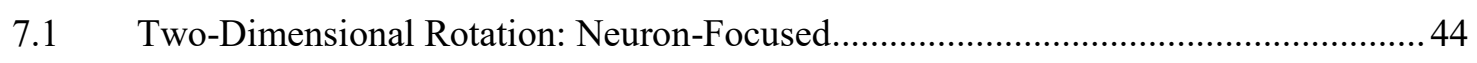

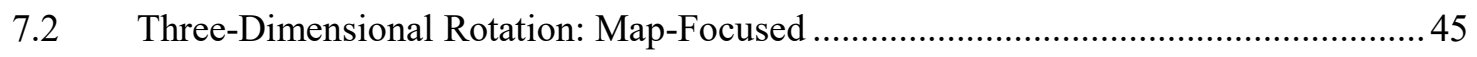

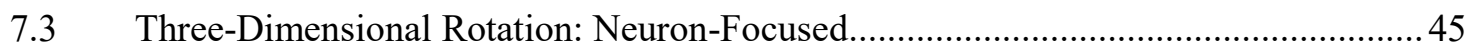

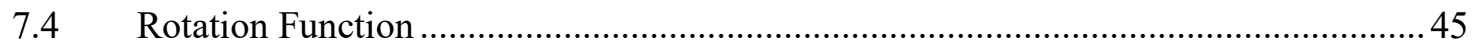

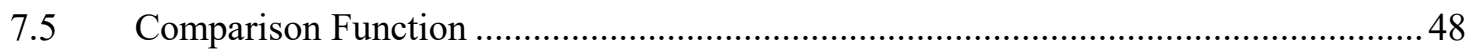

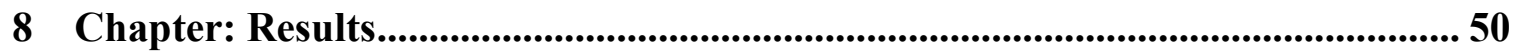

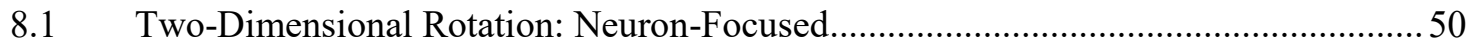

8.3 Three-Dimensional Rotation: Map-Focused …..................................................... 54

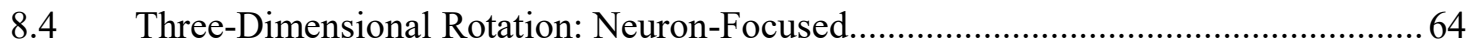

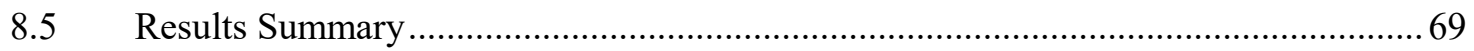

9 Chapter: Discussion ............................................................................................... 70

References ............................................................................................................................. 80 


\section{List of Tables}

Table 1: Expected vs. Actual Model Behaviour (Map-Focused) ................................... 54

Table 2: Expected vs Actual Model Behaviour (Neuron-Focused)............................... 64 


\section{List of Illustrations}

Figure 1: Cross-Section of Coordinate System.......................................................... 32

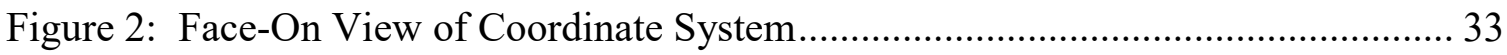

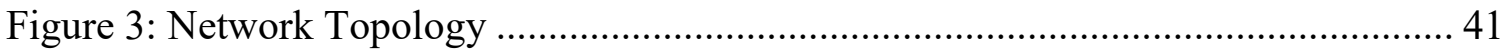

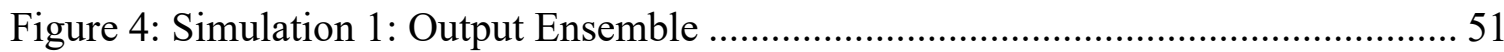

Figure 5: Simulation 1: Motor Monitor Ensemble …………………......................... 52

Figure 6: Simulation 2: Output Ensemble ………………............................................... 53

Figure 7: Simulation 3: Output Ensemble: 1 unit of separation .................................... 55

Figure 8: Simulation 3: SPP Transform Ensemble: 1 unit of separation ........................... 56

Figure 9: Simulation 4: Output Ensemble: 2 units of separation..................................... 57

Figure 10: Simulation 4: SPP Transform Ensemble: 2 units of separation ....................... 58

Figure 11: Simulation 5: Output Ensemble: 3 units of separation.................................... 59

Figure 12: Simulation 6: Output Ensemble: 4 units of separation.................................... 60

Figure 13: Simulation 7: Output Ensemble: 5 units of separation....................................... 61

Figure 14: Simulation 8: Output Ensemble: 6 units of separation................................... 62

Figure 15: Simulation 9: Output Ensemble: 7 units of separation...................................... 63

Figure 16: Simulation 10: Output Ensemble: 1 unit of separation ................................... 65

Figure 17: Simulation 10: SPP Transform Ensemble: 1 unit of separation ....................... 66

Figure 18: Simulation 11: Output Ensemble: 2 units of separation................................. 67

Figure 19: Simulation 12: Output Ensemble: 3 units of separation................................. 68

Figure 20: Transformation Network Acting on Representation Network ........................ 72 


\section{Chapter: Introduction}

Our ability to experience phenomena independent of external stimuli is, arguably, one of the hallmark features of the human condition; the fact a person can imagine complex objects and properties without needing any perceptual input for said objects and properties is as fascinating as it is impressive. Though it also raises some important questions, namely how these imaginings are represented, and how these representations are manipulated. It certainly feels as though these imaginings are similar to perception, with phrases such as, "the mind's eye" being commonplace in colloquial English, but the seemingly image-like nature of these imaginings may simply be epiphenomenal, with the underlying cognitive and neural mechanisms operating in a way that is far removed from the mechanisms of perception. Sadly, we have yet to uncover definitive answers to these questions, though as we will see, there is growing evidence suggesting these imaginings are, indeed, picture-like.

In the academic literature, these imaginings are often referred to as mental imagery: the presence of a perception-like representation of an entity, without perceptual input regarding that entity (Kosslyn, Thompson \& Ganis, 2006, p.4). The proceeding text will adopt this terminology, and will provide a general overview of the current state of the literature. The text will then focus on a specific mental imagery task (mental rotation), which will be followed by a discussion of how we can gain insight into mental rotation - and mental imagery more generally - with tools from computational neuroscience. The text will then outline a model of how mental rotation may occur at both the cognitive and neural level, which will segue into an overview of the model's results, followed by a discussion of how those results fit within the literature. 


\section{Chapter: Mental Imagery}

From Plato's Theaetetus to the memoirs of Sir Frances Galton (1880), mental imagery has long been an area of interest to academics across a broad spectrum of disciplines. These early investigations were typically philosophical in nature as the complex and abstract nature of mental imagery largely precluded it from the scientific method; however, the ever-maturing nature of scientific inquiry has combined with the rapid technological advances of the 20th Century to shift the modern study of mental imagery firmly into the domain of science. This has brought about a variety of competing theories, which can be roughly sorted into two frameworks: depictive and descriptive.

\subsection{Depictive Framework}

Largely popularized by Kosslyn, the depictive view posits that mental imagery is quasi-pictorial, and preserves metric distances (see Kosslyn, Thompson \& Ganis, 2006 for a detailed overview). This (mostly) falls in line with that we intuit when we think about mental imagery, and is the dominant view in the field, with $94 \%$ of psychologists who study mental imagery expressing their support for the depictive framework (Kosslyn, Thompson, \& Ganis, 2006, p. 179). However, despite the framework's similarity to our intuitions about mental imagery, depictive theories are quick to dismiss the notion that there are literal pictures in our minds (Kosslyn \& Pomerantz, 1977), instead suggesting that mental images take the same form as representations generated from perception, including both spatial and object properties (Kosslyn, Thompson \& Ganis, 2006, p. 4). 
With respect to this form, depictive theorists hold that mental images are comprised entirely of points and empty space, and that points can vary in terms of size, colour, and the density of their arrangement; moreover, each point in the mental image must correspond to a point in/on the actual object(s), with the distances between points in/on the actual object being preserved in the corresponding mental image (Kosslyn, Thompson \& Ganis, 2006, p. 11-12). What is important to note is that even though literal images satisfy all of the above criterion, they are not the only form that does. For example, the matrix

$$
\left[\begin{array}{ccc}
255 & 75 & 75 \\
75 & 255 & 75 \\
75 & 75 & 255
\end{array}\right]
$$

is said to be depictive as space is represented via the indexing of elements, with the properties of the object at each location in space being encoded in the value at its index (greyscale pixel values in this case). It should also be noted that the properties inherent to mental images are derived from their form (Pylyshyn, 2003, p. 289). For example, it will take longer to mentally scan across a wall than a picture hanging on said wall because one is larger than the other, both in the physical world and in mental imagery.

\subsection{Descriptive Framework}

In contrast to the depictive account, descriptive theories posit that cognition exists as some sort of mentalese that is language-like in form (Fodor, 1975). Although we do not know what this mentalese actually looks like, various attempts have been made to demonstrate the applicability of existing forms of descriptive representation to cognitive processes, including mental imagery.

For example, Davis (2011) demonstrated that first-order logic and a small number of IF-THEN rules are all that is needed to reason about the shape, location, properties, 
and movement of objects, even in the presence of incomplete information (Davis, Marcus \& Frazier-Logue, 2017). Given the pivotal role formal logic plays in cognitive processes such as language (e.g., Fox, 2007; Kamp \& Reyle, 2013), it is not unreasonable to tether our understanding of mental imagery to this form of representation. That said, the rigidity of formal logic - and the fact a single contradiction renders the entire system invalid - has led some to consider other forms of descriptive representation (Pylyshyn, 1973a).

One alternative is the use of graphical representations, such as Bayesian networks and their derivatives, which eschew the (largely) rigid nature of formal logic for the flexibility of conditional dependencies that describe the interrelations between variables (see Charniak, 1991 for an overview of Bayesian networks). A notable example of this form comes from Rosenbloom (2011), who built a factor graph that used mental imagery to solve The Eight Puzzle boards by first storing the board in working memory, then applying a set of conditionals that mentally shifted game pieces based on the state of the board. Another example can be found in the work of Schultheis and Barkowsky (2011), whose cognitive architecture, dubbed Casimir, combined semantic networks with analogical representations to model the mental processing of spatial knowledge. Overall, various graphical representations have proven useful in a number of different cognitive tasks, including knowledge representation and linguistics (e.g., Peters \& Shrobe, 2003; Stenning \& Oberlander, 1995); however, there are concerns regarding the extent to which such methods actually model cognition, or simply produce results that mirror those from psychological studies (see Grootswagers, Kookier \& Sloff, 2012). 
A second alternative can be found in rule-based - or, as some prefer, proceduralbased - systems (Pylyshyn, 1973b). Systems such as these have proven useful in problem solving and the design of expert system (e.g., Shortliffe, 2012), and operate in a manner similar to logic-based systems, only with rules (or procedures) replacing logical formulizations. When it comes to cognition as a whole, these rules are thought to be implicit, procedural, and exist as a structure of primitives built in response to stimuli (Pylyshyn, 1973b).

Across all of these forms, the unifying theme, insofar as they concern mental imagery, is the absence of depiction, and the notion that the properties of mental images exist independent of form, instead coming from a source external to the mental image, such as our prior experiences. In other words, it takes longer to scan a wall than a picture on said wall not because the mental images differ in size, but because we impose our knowledge of the physical world upon mental images.

\subsection{Behavioural Findings ${ }^{1}$}

Early-modern work attempted to hone in on the underlying form of mental imagery through a series of novel behavioural studies, such as the mental map scanning paradigm popularized by Kosslyn, Ball, and Reiser (1978). Here, participants were asked to memorize an island-shaped map with seven distinct, pictorial markers at various locations (e.g., a well, hut, tree, and-so-on). Once memorized, participants were given a location (verbally) and told to imagine its marker. After five seconds, they were verbally given a second location that might, or might not, be on the map. If the location was on the map, they were to mentally scan to said location by envisioning a black dot "zipping"

\footnotetext{
${ }^{1}$ Parts of this section have been taken from Riley and Davies (Forthcoming) with the authors' permission.
} 
across the map. When they saw the black dot reach the center of the target, they were to press a button. If the second location was not in the map, they were to respond with a different button press. Overall, Kosslyn and colleagues found that the time required to scan between two locations scaled linearly with the distance between them, and that distance and reaction time (RT) were correlated at 0.97 .

Since Kosslyn and colleagues landmark study, a wealth of converging evidence has yielded support for the depictive view of mental imagery. For example, Denis and Cocude (1988) replicated the map scanning paradigm, only they added a condition whereby maps were learned from verbal descriptions, not pictures. The results from this new condition mirrored those from visual inputs (linear RTs), thus suggesting that the space-preserving nature of mental images is/was not dependent on visual perception. This conclusion is/was strengthened by the work of Röder and Rösler (1998), who had 51 sighted and 19 congenitally blind participants examine maps visually or haptically (or both), and then mentally scan as per Kosslyn and colleagues original study. Once again, linear RTs were found across all combinations of sightedness and modality (except, of course, congenitally blind and visual modality, which was not investigated for the obvious reasons).

These conclusions did not go unchallenged, however, with some claiming that the linear RTs were a function of the task instructions, not properties inherent to mental images. One counter-study by Pylyshyn (1981) used the same map scanning paradigm, only participants were also told there was a light source at each location, and a switch that could turn the source on or off. Here, participants were told to focus on a specific location, then told to imagine that the switch for a second location was flipped, and to 
respond when they saw the corresponding light turn on/off. Unlike Kosslyn and colleagues' original study, RTs were not a function of the distance between the two locations, which lead to the conclusion that mental scanning was cognitively penetrable, and that the underlying representation did not preserve metric distances. The work of Pylyshyn - and, more generally, the view that mental imagery is largely dependent on a person's tacit knowledge of the world - has been supported by a number of different studies (e.g., Goldstone, Hinrichs \& Richman 1985), sowing further discord into the debate. In truth, we may not be capable of identifying mental imagery's underlying form via behavioural studies (Anderson, 1978), though recent modelling efforts may help shed some light on these divergent map scanning results (see Riley \& Davies, Forthcoming).

\subsection{Neuroscience Findings}

The seeming failure of behavioural studies to yield a definitive conclusion regarding the underlying form of mental imagery has shifted much of the current debate into the domain of neuroscience, the thinking being that the underlying neural mechanism will tell us how the mind operates.

\subsubsection{Topological Organization of the Primary Visual Cortex}

Some of the most compelling evidence that mental imagery is depictive can be found in the work of Tootell and colleagues (1982). Here, Macaque monkeys were anesthetized and shown a log-polar pattern of blinking lights. They were subsequently injected with a radioactive dye that stained activated neurons, then sacrificed and dissected. Upon inspecting V1 in the visual cortex, it was found that the dye had stained the cortex in a matter that closely resembled the spatial properties of the original light pattern, which led to the conclusion that space on the cortex was, quite literally, used to 
represent space in the physical world. Of course, topological organization in Macaque monkeys does not entail topological organization in humans; however, efforts to map both the human and Macaque visual cortex suggests there is a high level of interspecies overlap in more primitive areas of the visual cortex, such as V1, with less interspecies overlap in areas farther away from V1, notably the extrastriate visual cortex (Van Essen et al., 2001). Moreover, there is also fMRI evidence that retinotopic stimuli, such as the blinking light pattern used in Tootell et al. (1982), does not engender activity in the extrastriate visual cortex, and that this stimuli produces activation in V1 and V2 that is consistent with the notion of topological organization (Tootell et al., 1998). Taken as a whole, it seems that primary visual areas such as V1 are likely to be topologically organized in both Macaque monkeys and humans, and that if mental imagery is to be viewed as depictive, we should see increased activity in these brain regions during both perception and imagery.

\subsubsection{Shared Correlates of Visual Perception and Mental Imagery}

To this end, Kosslyn et al. (1995) used positron emission tomography (PET) to scan participants' brains while they imagined common objects such as a telephone or clock, and while they imagined nothing but black. Overall, it was found that small mental images ( 0.25 degrees of visual angle) produced greater activation in the posterior part of the medial occipital region of the visual cortex (an area that activates during the perception of small images), and that large mental images (16.0 degrees of visual angle) produced greater activation in the anterior part of the medial occipital region (an area that activates during the perception of large images). That being said, these findings are not always replicable (e.g., Markus et al., 2000); however, a meta-analysis by Kosslyn and 
Thompson (2003) demonstrated that these replication issues can largely be traced to methodological factors that may not elicit the formation of visual mental images. Still, some have argued that activation of early visual areas is not sufficient, and if one is to support a depictive view of mental imagery, activation in these regions needs to reflect the spatial properties of the image (Pylyshyn 2002).

To address this, Slotnick, Thompson, and Kosslyn (2005) used event-related functional magnetic resonance imaging (fMRI) to construct retinotopic maps in three conditions: perception, imagery, and attention. In the perception condition, checkerboard wedges rotated about a fixed point, with red squares periodically appearing on the screen. The stimuli produced a retinotopic map in the visual cortex, with participants responding whether the appearance of a red square was inside, or outside, the wedges. In the imagery condition, the same red square procedure was administered, but with only the outer edges of the wedges being shown, thus forcing participants to imagine the missing aspects of the original stimulus. Finally, in the attention condition, only the outer edges of the wedges were shown, except participants were explicitly told not to imagine the missing aspects of the stimulus, and to respond by indicating what side of the screen the red squares were on. Overall, it was found that the isolated effects of the imagery condition [(imagery + attention $)$ - attention] produced increased activity in topologically organized areas of the visual cortex, and that activation under imagery was similar to activation under perception, thus suggesting that visual mental imagery preserves spatial properties.

\subsubsection{Visual Imagery vs. Spatial Imagery}


All that being said, there is evidence of a divide between visual mental imagery (or what we would call, "the mind's eye") and spatial mental imagery (where objects are located in space). For example, a brain-damaged patient with severe impairments in visual recognition was able to successfully complete a battery of spatial imagery tasks, such as mental rotation, but was significantly hindered during a battery of visual imagery tasks, such as size comparison (Farah et al., 1988). This divide is further supported by neuroimaging studies, including Mellet and colleagues (1996), who found no activation in early visual areas associated with visual imagery when participants were told to mentally construct cube assemblies from verbal descriptions. However, there also appears to be a divide within spatial imagery itself, with location and transformation components relying on different brain networks (Thompson et al., 2009). This falls in line with the depictive view that information regarding location and orientation are stored in a single spatial map, and that transformations to this map occur when the map is passed to more posterior parts of the cortex (Kosslyn, 1996; Thompson et al., 2009).

\subsection{Criticisms of Depictive Theories}

Despite the aforementioned evidence for depictive mental imagery, there are some genuine reasons to be uncomfortable with the notion. For example, it has been argued that the matrix structure advocated for by depictive theorists is a functional space that has no intrinsic properties; it is the way in which we understand said matrix that bestows upon it spatial properties (Pylyshyn, 2003, p. 360-361). As Pylyshyn (2003) points out, spatial concepts such as "adjacent" only apply to matrices when they are placed within an actual space, and that independent of said space, a matrix consists entirely of symbols (p. 363). Otherwise stated, it is easy to move from one location in the 
matrix to any other location within the matrix, and any attempt to restrict movement within the matrix must come from an external source independent of the matrix itself (Pylyshyn, 2003, p. 364-365). From this, it has been argued that a matrix structure reflects a form of descriptive adequacy that sufficiently describes the behaviour of mental imagery, but not explanatory accuracy regarding mental imagery’s underlying form (Pylyshyn, 2003, p. 362).

There are also questions surrounding the role of neuroscience in the imagery debate. For example, much has been made of Kosslyn et al.'s (1995) work demonstrating that small and large mental image evoke activation in brain regions corresponding to the perception of small and large images. However, what this work does not take into account is why these regions activate during perception, which in this instance is because of how fibers from the periphery of the retina connect to the occipital cortex (Pylyshyn, 2003, p. 395). In other words, if the perception of large and small images evokes activation in different brain regions due to retinal fibers and not properties inherent to the images themselves, then one can not claim that the activation of these regions under imagery necessitates a depictive account of mental imagery. This argument is partially undercut by the work of Slotnick, Thompson, and Kosslyn (2005) (see section 2.4.2); however, the question of whether neuroscience deserves a privileged role in the imagery debate remains to be seen [e.g., even if a monitor-like display was found on the cortex, the fact many imagery tasks are cognitively penetrable brings into question the role this display plays (Pylyshyn, 2003, p. 388)].

\subsection{Rebuttal by Depictive Theorists}


To address the matrix issues raised by Pylyshyn (2003), depictive theorists point out that individuals can be looking in one direction, but direct their attention in another; thus to scan a matrix, all one needs to do is shift their attention as needed, not move through a physical space along a specific trajectory (Kosslyn, Thompson \& Ganis, 2006, p. 40). Moreover, they also note that a matrix structure can be depictive without being embedded in a coordinate system as this structure implicitly conveys geometric information such as size (Kosslyn, Thompson \& Ganis, 2006, p. 41). Kosslyn, Thompson \& Ganis (2006) also remark that, "representations only 'represent' within the context of a processing system" (p. 50). In the case of the human brain, what matters is that neural connections process the representation in a way that preserves space; although this processing can occur via any number of cortical activation patterns, it just so happens that these activation patterns use space on the cortex to represent space in the physical world (Kosslyn, Thompson \& Ganis, 2006, p. 50-51). Overall, the debate whether a matrix constitutes a depiction is nuanced and far from settled, though both sides agree that mental imagery can be thought of in terms of this data structure.

Remarks have also been made in response to claims that neuroscience evidence should not be given a privileged role in understanding mental imagery. At first glance, the purported cognitive penetrability of many imagery tasks does bring into question what role a "cortical depiction" plays in mental imagery; that is, if mental imagery is largely dictated by tacit knowledge, then such a depiction will be irrelevant. However, it is argued that this claim relies on perception being driven entirely by bottom-up processes (Kosslyn, Thompson \& Ganis, 2006, p. 68). In actuality, perception is driven by both bottom-up and top-down processes, with top-down processes altering cognitive 
and neural processing according to one's knowledge and/or beliefs (Kosslyn, Thompson \& Ganis, 2006, p. 68). This, again, is a nuanced but important distinction: cognitive penetrability necessitates that phenomenon be altered by one's knowledge and/or beliefs about that phenomenon, whereas top-down processing can alter processing so-as-to incorporate knowledge and/or beliefs without altering the phenomenon (Kosslyn, Thompson \& Ganis, 2006, p. 68). In any case, the debate over the role of neuroscience can, in many ways, be summarized as a debate over whether "the mind is what the brain does" (Kosslyn, Thompson \& Ganis, 2006, p. 134). For proponents of depictive theories, the human brain and its connections provide both a framework and a set of constraints for formulating theories of cognition (Kosslyn, Thompson \& Ganis, 2006, p. 134). Thus, any attempt at understanding cognitive processes should begin with an understanding of neural activation during those processes.

Overall, this research views mental imagery as depictive, but acknowledges the imagery debate is far from settled.

\subsection{Kosslyn's Theory of Mental Imagery}

In their book The Case for Mental Imagery, Kosslyn, Thompson, and Ganis (2006) lay out what is largely considered to be the preeminent theory of mental imagery. As depictivists, they view mental imagery as complex, analogue data structures that arise from representations in long-term memory, and that operate in a manner that is similar to visual perception. With respect to this visual component, a visual buffer is thought to reside in the topologically organized areas of the primary visual cortex (PVC), and contain a hybrid representation whereby each point in these areas not only represents the presence/absence of a point in space, but also the properties inherent to objects (e.g. 
colour, depth, motion, and-so-on) via a propositional code (p. 135). Moreover, the representation within this visual buffer is not complete, and the presence or absence of information within the representation depends on whether or not that information has been selected by the "attention window," which itself can be shifted to either (i) new locations in space, or (ii) different objects (p. 135-136). Finally, the visual buffer does not convey pictures in the traditional sense, but rather information that can be further processed (p. 136).

In terms of this additional processing, a "spatial-properties-processing subsystem" (SPP) and an "object-properties-processing subsystem" (OPP) are thought to manage spatial and visual imagery, respectively (p. 137). In the case of the OPP, it takes in information from the attention window and extracts properties such as shape, colour, andso-on, then determines if the object in question is familiar (p. 137). Similarly, the SPP also take in information from the attention window, only it analyzes said information for spatial properties such as location, size, orientation, and-so-on, then builds an "object map," (also referred to as a "spatial map") which, as the name implies, is simply a map of where object are located within the space of the visual field (p. 137-138). Moreover, spatial imagery and the SPP have two distinct components: a location component that positions an object in space, and a transformation component that moves an object within space (p. 137-138).

Once the contents of the visual buffer have been processed by the SPP and the OPP, their outputs are sent to associative memory and compared against stored representations; if a match is found, a description of the information in the visual buffer is formed from the matching, stored representation (p. 139). If no match is found, then the 
"best-matching" representation is used, though it is treated as a tentative hypothesis about what the object/scene could be (p. 139). When this occurs, an "information shunting system" (ISS) is activated, with the ISS serving as a bridge to other cognitive subsystems so-as-to shift the attention window to unique regions that may assist in object/scene identification (p. 139-140).

When a person forms a mental image, they first go into long-term associative memory to retrieve a spatial layout (p. 143); if this spatial layout is all the task requires, then the stored information is used to create a spatial map in the SPP. However, if properties (such as colour, texture, and so on) are required, then the ISS goes into longterm associative memory and retrieves the required property(ies), then sends that information to the OPP, which then sends it backwards to the visual buffer, ultimately resulting in the formation of a visual mental image (p. 144). However, unlike visual perception, depictions in the visual buffer fade quickly, which means maintaining the depiction requires repeated re-activations of the OPP, which then re-sends the information back to the visual buffer (p. 145).

Overall, Kosslyn and colleagues' theory serves as a guide for my model, namely the existence of a SPP that delineates between representation and transformation, and that spatial maps do not encode object properties. 


\section{Chapter: Mental Rotation}

Given the depth and complexity of mental imagery research, it is difficult to understand in its entirety, let alone model computationally. As such, we, as researchers, are behooved to narrow our focus to specific imagery tasks, slowly building up a broader understanding piece-by-piece. To this end, I have elected to focus my attention on mental rotation as it is a well documental imagery task with a (reasonably) clear pattern of results.

\subsection{Behavioural Findings}

In what is arguably one of cognitive science's most influential papers, Shepard and Metzler (1971) showed participants multiple pairs of irregularly shaped, three dimensional cubes assemblies projected into two dimensions, and asked if the two images were mirrors of each other, or if one was a rotation of the other. Surprisingly, they found that participant RTs for identifying rotations were directly proportional to the difference in angular rotation. To explain this phenomenon, Shepard and Metzler proposed that participants used picture-like mental imagery to form a three dimensional image of the cube, then rotated that mental image as a whole unit. In other words, the linear RTs were thought to be a function of the properties inherent to mental images, not one's knowledge of the physical world.

Importantly, there is evidence that mental rotation is a spatial imagery task that does not depend on the visual system. For example, Marmor and Zaback (1976) replicated Shepard and Metzler's original study with haptic images presented to both blind and sighted but blindfolded participants. Overall, they were able to re-produce the linear RTs from Shepard and Metzler's original study - despite the lack of visual input - 
and although the majority of the participants self-reported the use of mental rotation ( $94 \%$ of the blind and $69 \%$ of the blindfolded participants), five participants self-reported the use of logical rules. There are, of course, issues with self-reports, though the results do evoke question regarding the ubiquity of rotation as a task strategy, and whether mental rotation is cognitively penetrable.

To this end, Cooper (1976) showed participants an angular shape and had them mentally rotate it clockwise. During the mental rotation, participants were shown the angular shape again, only in a rotated position, or in a reflected position. Participants responded with whether the image was the original angular shape, or a reflection of the original angular shape. Overall, Cooper (1976) found no differences in RTs when the image was presented in an orientation that was thought to coincide with the orientation of the participant's mental rotation (referred to as familiar orientations, and calculated from Shepard and Metzler's original study), nor were there any differences between RTs for familiar and unfamiliar orientations. To explain these findings, Cooper (1976) proposed that participants performed a continuous rotation as per Shepard and Metzler (1971). Here, it was thought that a continuous rotation passing through a series of orientations prepared participants for the presentation of an image in an unfamiliar orientation; if some other strategy was used to perform the task, then one would expect that familiar orientations would produce faster RTs than unfamiliar orientations (Cooper, 1976).

However, there is also evidence that a "whole unit" rotation may not always occur. In one key study, Bethell-Fox and Shepard (1988) showed that although a large majority of participants used mental rotation as a task strategy, the complexity of the stimulus determined the nature of said rotation. That is, complex and unfamiliar stimuli 
resulted in a piecewise rotation where individual parts were rotated into place one-byone, with less complex stimuli being rotated as a whole unit (Bethell \& Shepard, 1988). Moreover, the authors also demonstrated that, given sufficient engagement, participants could become familiar enough with complex stimuli to rotate them as a whole unit (Bethell and Shepard, 1988). In a related vein, work by Tarr and Pinker (1989) demonstrated that people hold in memory a series of stored views/orientations that objects are rotated into/out-of alignment with. This multiple-views hypothesis has been well replicated (see Tarr, 1995), and suggests that familiar objects have more stored orientations that can be leveraged during rotations, at least compared to unfamiliar objects.

When it comes to cognitive penetrability, Borst et al. (2011) conducted two experiments supporting the notion that mental rotation is not cognitively penetrable. In the first experiment, they placed participants into one of two rotation conditions: the Shepard and Metzler paradigm, or one that had participants identify misoriented alphanumeric characters (Borst et al., 2011). Within each rotation condition, participants were given one of three different task strategies: a mental rotation strategy, a leap condition that had participants imagine the object perform a single "jump" to the desired orientation, or an analytic condition that had participants compare various parts of the objects when completing the task (Borst et al., 2011).

In the Shepard and Metzler condition, not only did RTs increase alongside angular disparity in all of the three instruction conditions, the slopes (and intercepts) of RTs, as well as the error rates, did not differ across all instruction conditions, thus suggesting that the rate of rotation across all three groups did not differ, which runs 
counter to the tacit knowledge prediction of faster RTs and a slope near zero when under the leap instruction condition (Borst et al., 2011).

In the alphanumeric characters condition, there were no significant differences between the instruction sets; however, there was a trend (faster RTs) for the leap condition (Borst et al., 2011). That said, a large probability of Type-II error was observed, the RTs within the leap condition were still linear, and there was a higher error rate relative to the other instruction sets. Given this, the authors postulated that participants in the leap condition were still performing a rotation that passed through intermediary points, only they were attempting to do so at a faster rate, thus leading to more errors.

To explore the alphanumeric character results further, a second, repeated measures experiment was leveraged in an attempt to directly manipulate participant's tacit knowledge of rotation speed. First, the Shepard and Metzler paradigm was repeated to get a baseline, then participants were shown a video of the object rotating clockwise or counterclockwise. Half of the videos showed the object rotating twice as quickly as the average rotation speed in the first experiment, with the remaining videos showing rotations at half speed. Participants were explicitly told to pay attention to the rate of rotation as they would be performing a mental rotation task with those objects (Borst et al., 2011). The Shepard and Metzler paradigm was then administered a second time.

Overall, RT slopes for the two video conditions did not vary across the first or second iteration of the rotation task. That said, the second iteration of the task produced lower intercepts for the $2 \mathrm{x}$ video condition than the $0.5 \mathrm{x}$ video condition. Taken together, the authors concluded that participants were able to delay or speed up their 
overall RTs (intercepts), but were not able to alter the speed of rotation (slopes). When considered alongside the results of experiment one, the authors proposed that rotation speed is constrained by the underlying mental representation, and that mental rotation is not influenced by tacit knowledge, nor is it cognitively penetrable.

In sum, these behavioural findings suggest that mental rotation is a spatial imagery task that operates independent of the visual system; it does not appear to be cognitively penetrable, nor influenced by tacit knowledge. On the whole, when confronted with spatial imagery tasks, the overwhelming majority of people employ rotation as a strategy; however, the nature of this rotation is moderated by stimulus complexity: complex and unfamiliar stimuli seem to elicit a piecewise rotation, with simple and complex-but-familiar stimuli a whole unit rotation. Moreover, people appear to hold multiple orientations of familiar objects in memory, though the rate at which any orientation can be rotated appears to be constrained, possibly by the underlying form of the mental image.

\subsection{Neuroscience Findings}

Taking a bird's eye view, a meta-analysis of 32 PET and fMRI studies revealed that mental rotation engenders increased activity in topologically organized areas of the visual cortex, such as the intraparietal sulcus, as well as areas linked to motor simulation, such as the medial superior precentral cortex (Zacks, 2008). To explain this pattern of results, Zacks proposed that mental rotation involves continuously updating the relationship between an object-centered frame of reference and an environment-centered (also referred to as allocentric) frame of reference. However, the larger take-away is that these results not only provide further support for depictive mental imagery, but also 
suggest that motor processes play a role in transformations applied to spatial maps (also see Wrage et al., 2002), which helps corroborate Kosslyn, Thompson, and Ganis’ (2006) view of a demarcation between spatial representations and spatial transformations.

That being said, the interplay between frames of reference and mental rotation is more convoluted than is suggested by the findings of Zacks (2008). For example, Hegarty and Waller (2004) conducted a series of behavioural studies demonstrating that mental rotation and perspective taking are unique, but highly correlated, mental abilities, and proposed that this dissociation is a byproduct of differences in people's ability to make egocentric and object-centered transformations. Similarly, Just and Carpenter (1985) showed that although a coordinate system based on an egocentric frame of reference was dominate in mental rotation tasks, other coordinate systems can be used to successful rotate objects.

Turning back to neuroscience, a thorough review by Filimon (2015) suggests that all spatial reference frames are egocentric. This is certainly a minority opinion, though there are others who question the status of allocentric maps (see Ekstron, Arnold \& Iaria, 2014), as well as notable findings that give this egocentric theory credence: Place, grid, and head direction cells, which have traditionally been thought to form allocentric maps of the environment (e.g., McNaughton et al., 2006; Moser et al., 2008), may actually form egocentric maps as the activity of (at least some) cells appear to depend on behaviour and the amount of sensory information received, despite responding to the same location in space (Ulanovsky \& Moss, 2011). This notion is further supported by research suggesting that spatial representations are dependent on behavioural goals, as evidenced by an over-representation of place fields around salient locations (Hollup et al., 
2001), as well as evidence that some of these cells are re-activated during (supposed) animal dreams/memories involving known locations (Pavlides \& Winston, 1989). As such, Filimon (2015) proposes that place cells signal place recognition; that grid cells generate egocentric maps as grid cells are too rigid to produce allocentric maps, which are highly flexible; and that instead of comparing the orientation of external objects to a reference point/landmark, head direction cells compare an egocentric orientation with an external point/landmark. At a minimum, there is evidence the visual system utilizes egocentric coordinates (Tarr \& Pinker, 1990), which suggests mental imagery will as well.

Overall, the evidence supporting Kosslyn, Thompson, and Ganis' (2006) proposed divided between spatial representation and transformation leads one to suspect Zacks' (2008) view that mental rotation involves updating frames of reference is at least partially correct; however, it also appears that the frames of reference in question may not be object-centered and environment-centered. Instead, this thesis takes the view that mental rotation involves spatial maps that are viewed through an egocentric frame of reference, with the map being updated according to a "motor monitor" that continuously instructs transformations, which in the case of rotation is movement away from a familiar orientation. Moreover, this thesis will attempt to replicate part of the Shepard and Metzler (1971) paradigm by building a network that can (i) mentally rotate a series of connected voxels (hereafter referred to as cube assemblies) that are similar to the stimuli from the Shepard and Metzler (1971) paradigm (I lack the resources for exact stimuli replication), and (ii) determine when it is in alignment with a second cube assembly (I am omitting the reflection aspect of the task as I am explicitly interested in the act of mental 
rotation, no-so-much the comparison component). Finally, these cube assemblies are thought to be simple due to their limited number of voxels, but unfamiliar due to their artificiality. Thus, they will engender a continuous, whole unit rotation away from the familiar, canonical orientation. 


\section{Chapter: Model Representation}

With the aforementioned knowledge of mental imagery and mental rotation, the (arguably) biggest consideration that remains is determining what coordinate system the spatial map is to reside in. Turning to biology, there is evidence that the retinas of some vertebrates possess a polar coordinate system whereby a series of concentric circles specify the radial component (Grant \& Rubin, 1980; MacDonald, 1977), as well as evidence that the metric distortions found in Tootell and colleagues' (1982) monkeys can be replicated via an isotropic mapping of Cartesian retina coordinates to polar cortex coordinates (Schwartz, 1984); however, the extent to which these findings apply to humans is not fully understood. That said, there is notable evidence that a polar coordinate system is the most computationally efficient (e.g., Longuet-Higgins \& Prazdny, 1980; Tistarelli \& Sandini, 1993), and that polar coordinates best explain a variety of neurophysiological and psychophysical data (Zetzsche, Krieger \& Wegmann, 1999). Overall, the current research takes the view that spatial maps reside in a polar coordinate system that is discretized according to visual acuity; that is, each discrete section in the spatial map represents the smallest perceptible area (see Figures $1 \& 2$ ). 


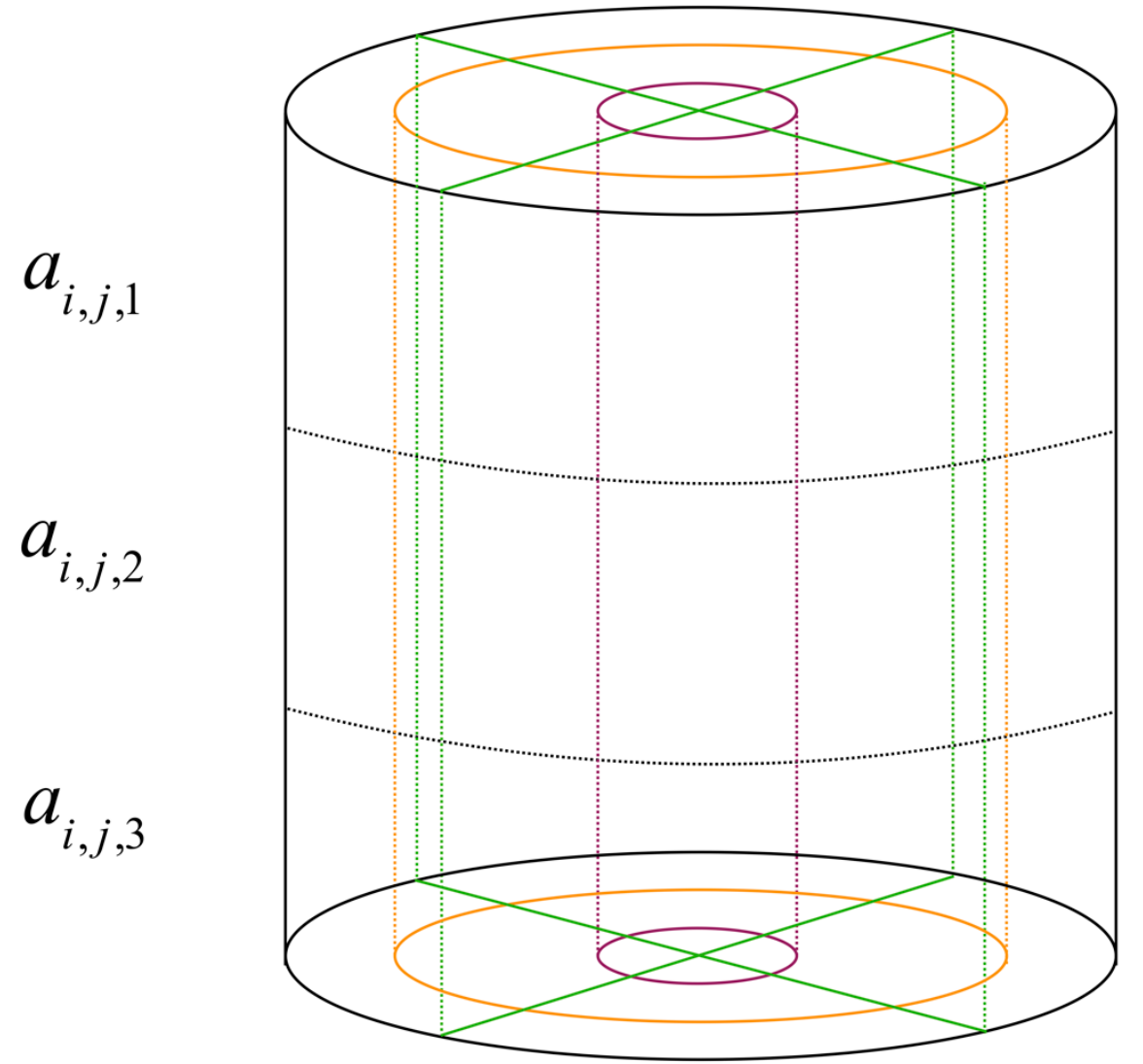

Figure 1: Cross-Section of Coordinate System

A cylindrical coordinate system comprised of concentric circles is used to construct spatial maps. Solid black, purple, and orange denote concentric circles that discretize the radial component. Solid green denotes planes that discretize the angular component (this image is divided into quarters for clarity, but any number of planes can be added/subtracted, as can any number of circles). Dashed black lines denote boundaries that discretize depth. Dashed purple, orange, and green lines show the inner boundaries of their respective circles/planes. Each voxel in the coordinate system is given by $\boldsymbol{a}_{\boldsymbol{i}, \boldsymbol{j}, \boldsymbol{k}}$ where $a$ denotes the presence/absence of a point (1/-1) within that voxel, $i$ the section within the circle, $j$ the circle itself, and $k$ the depth section (see Figure 2). This thesis limits maps to three depth sections. 


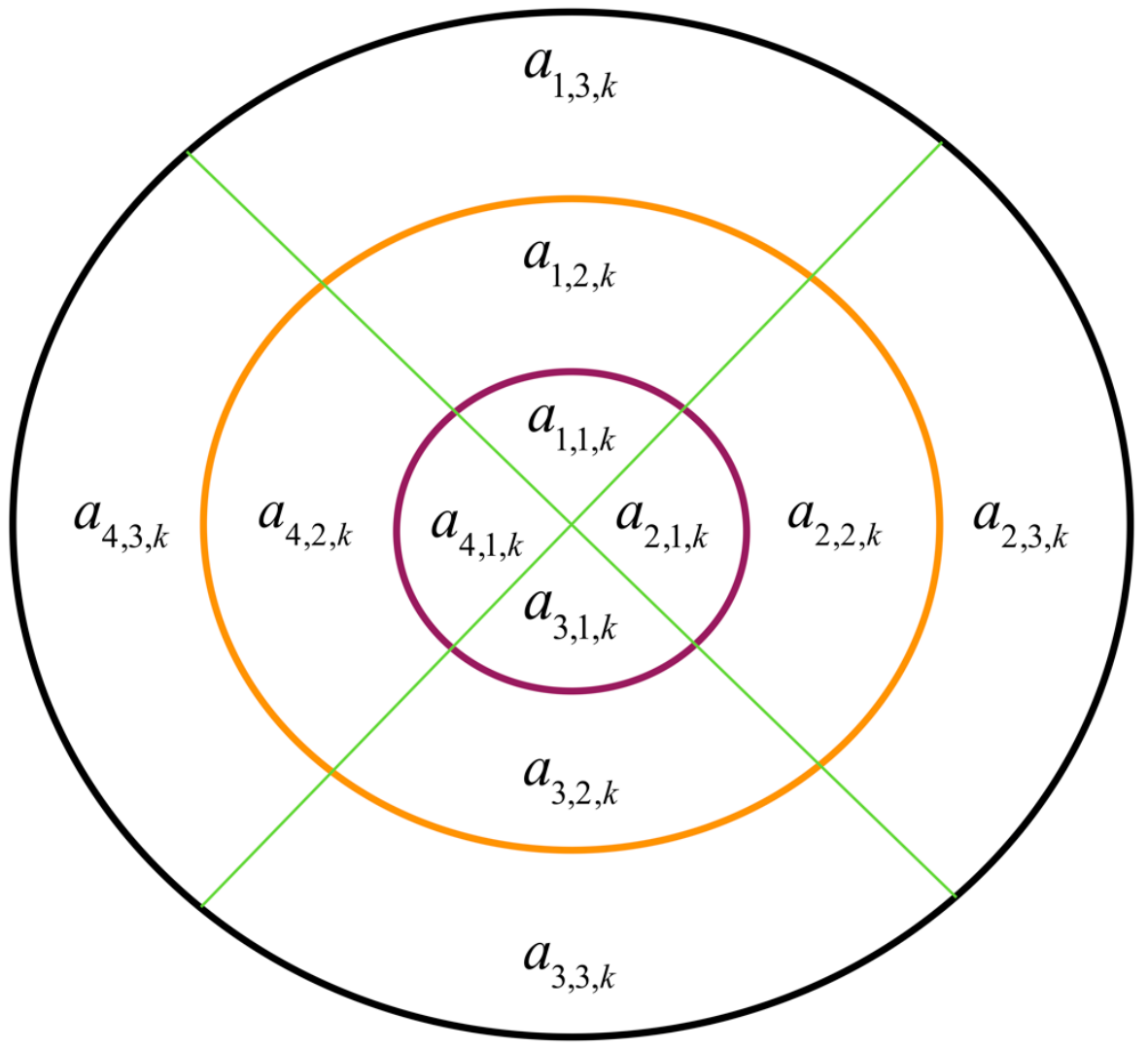

Figure 2: Face-On View of Coordinate System

Black, purple, and orange denote concentric circles that discretize the radial component. Green denotes planes that discretize the angular component (this image is divided into quarters for clarity, but any number of planes can be added/subtracted, as can any number of circles). Each voxel in the coordinate system is given by $\boldsymbol{a}_{\boldsymbol{i}, \boldsymbol{j}, \boldsymbol{k}}$ where $a$ denotes the presence/absence of a point (1/-1) in that voxel, $i$ the section within the circle, $j$ the circle itself, and $k$ the depth section. This thesis limits maps to three depth sections. 
Here, each circle (purple, orange, and black) is discretized into $i$ sections. Thus, for each circle, we can construct the vector $\boldsymbol{\psi}_{j, k}=\left[\begin{array}{lll}a_{1, j, k} & \ldots & a_{i, j, k}\end{array}\right]$ whose elements give us the presence/absence of a point at each location. The vector for each circle in each depth section can then be used to construct the spatial map

$$
\mathbf{M}=\left[\begin{array}{ccc}
\boldsymbol{\psi}_{1,1} & \cdots & \boldsymbol{\psi}_{j, 1} \\
\vdots & \ddots & \vdots \\
\boldsymbol{\psi}_{1, k} & \cdots & \boldsymbol{\psi}_{j, k}
\end{array}\right]
$$

where each row is a depth section and each column a circle. Importantly, depiction only requires that $\mathbf{M}$ be processed in such a way that $\mathbf{M}$ preserves space during said processing. This means other arrangements of the elements in $\mathbf{M}$ can be valid depictions, such as its transpose or vectorization, provided these arrangements are processed appropriately (see sections 2.6 and 7.4 ).

Moreover, this research only concerns itself with the representation and transformation of single objects, and operates under the pretense that the attention centers this object within the aforementioned spatial map. That said, an extended version of this map could be used to describe multi-object scenes, though it is beyond the purview of this thesis. To achieve this, I would describe scenes in terms of a larger, allocentric map with Cartesian coordinates $\left(\mathbf{M}^{+}\right)$; then, when attention is shifted to a location in this allocentric map, the object at that location would be centered within $\mathbf{M}$ via the attention window. Thus, by my thinking, $\mathbf{M}$ is a byproduct of the attention window, and is utilized when attending to a specific object(s) within $\mathbf{M}^{+}$. Moreover, $\mathbf{M}$ exists as a connected set of voxels (Figures $1 \&$ 2), with objects in $\mathbf{M}$ being a collection of voxels that have points present at their respective locations. 


\section{Chapter: The Neural Engineering Framework}

Our spatial map in hand, the next step is to find a tool that will allow us to build a biologically plausible model of mental rotation. To this end, I have elected to leverage the Neural Engineering Framework (NEF) and its associated Python library, Nengo (Bekolay et al., 2014) ${ }^{2}$. There are alternative tools I could have selected, such as Brian (Goodman \& Brette, 2009); however, Nengo's focus is on how cognition may stem from the underlying biology - which is a marked departure from the low-level biology simulations that drive tools like Brian (Bekolay et al., 2014; Goodman \& Brette, 2009) and thus is the ideal tool for building neural models of high-level cognitive functions, including mental rotation. In fact, Nengo has already been used to build the world's largest functional brain model (Eliasmith et al., 2012), as well as model a variety of other cognitive tasks, such as working memory (Singh \& Eliasmith, 2006), inductive reasoning (Rasmussen \& Eliasmith, 2014), and motor control (DeWolf \& Eliasmith, 2011), just to name a few.

\subsection{NEF Representation}

At its core, the NEF is driven by three basic principles, the first being representation, which focuses on how information can be encoded into time-varying patterns of neural activity (spike trains/rasters), and how these patterns can be decoded back into their original form (Bekolay et al., 2014). That is, representation focuses on how information can be translated between the state-space of the input and the neuronspace of the brain, the argument being that if the information encoded into the neuron-

\footnotetext{
2 Nengo contains two components: NEF and SPA (semantic pointer architecture). This thesis only uses NEF as SPA is concerned with symbolic processing.
} 
space was incapable of being decoded, then downstream brain regions would not be able to use that information in any meaningful way (Eliasmith, 2013, p. 46-51).

Conceptually, we can understand representation by supposing I only have three neurons. Let us further suppose that one neuron's tuning curve is such that its level of activation is dependent on an object's position along the $\mathrm{x}$-axis of my visual field; when an object is in the rightmost spot of my visual field, the neuron fires at $100 \%$, and as the object moves from right to left, the neuron's activation decreases proportional to the object's movement. Thus, when the object is centered in my visual field, the neuron will fire at $50 \%$, and when it is at the leftmost spot, it will fire at $0 \%$. Let neurons two and three exhibit the same properties along the y-axis and z-axis, respectively. Now, suppose an electrode is placed in each of my neurons, and that I am seated in a pitch-black room with my head and eyes secured so they can not move. Outside is a button that will release a firefly into the darkened room, and a machine that prints out the activation of each of my three neurons. When the button is pressed and the firefly released, the machine sends a constant stream of neural data. With this data and knowledge of my neurons' tuning curves, one could decode my pattern of neural activity and map the movements of the firefly about the darkened room. Thus, it can be said that each of neuron's activation patterns is an encoding of external information: the firefly's movements along the $\mathrm{x}, \mathrm{y}$, and $\mathrm{z}$ axes.

More formally, the NEF represents inputs as scalars or vectors, and utilizes a nonlinear encoding process whereby current is injected into neurons based on their tuning curves, which then causes the neurons to spike (Bekolay et al., 2014). Moreover, for each neuron, its tuning curve is determined by the neuron's gain, bias, and an encoding 
weight that specifies what input direction (positive or negative) leads the neuron to be most active (Bekolay et al., 2014). Computationally, encoding is given by

$$
\delta\left(t-t_{i m}\right)=G_{i}\left[\alpha_{i}\left\langle\mathbf{x e}_{i}\right\rangle+J_{i}^{\text {bias }}\right] \quad \text { (eq. 1) }
$$

where $\delta(t)$ is the neuron spike, $i$ the neuron index, $m$ the spike index, $G_{i}$ the neuron's response function, $\langle\cdot\rangle$ the dot product, $\alpha$ the gain, $\mathbf{x}$ the state-space being encoded, $\mathbf{e}$ the vector of encoding weights, and $J^{\text {bias }}$ the background current (Eliasmith, 2013, p. 396).

When it comes to decoding, spike trains are passed through an exponentially decaying filter to account for the generation of a postsynaptic current; each spike train in the newly filtered signal is then given a weight (derived from a least-squares solver that minimizes the representational error of the spike train), with the sum of those weighted activations producing an estimate of the original input (Bekolay et al., 2014). This process is given by

$$
\left.\widehat{\mathbf{x}}=\sum_{i, m}^{N, M} h_{i}\left(t-t_{i m}\right) \mathbf{d}_{i} \quad \text { (eq. } 2\right)
$$

where $\hat{\mathbf{x}}$ is the estimate of the original input; $N$ and $M$ the number of neurons and spikes, respectively; $i$ and $m$ the neuron and spike indexes; $\mathbf{d}_{i}$ the decoder for neuron $i$; and $h_{i}(t)$ the postsynaptic current, which is derived from $h(t)=e^{-t / \tau_{P S C}}$ where $\tau_{P S C}$ is the time constant of decay (Eliasmith, 2013, p. 397).

\subsection{NEF Transformation}

Though representation is, in-and-of itself, an impressive feat, it is of little use if we cannot transform the signal as it propagates between neurons. Given that decoding centers on extracting weights that minimize the representational error, if we want to decode some transformation of the signal, all we have to do is find a set of decoding weights that minimize the representational error of the transformation. 
For example, suppose we have two groups of neurons, $A$ and $B$; a scalar for the state-space, $x=2$; and a transformation we want to compute, $f(x)=x^{2}$. To compute this transformation, we would send $x$ into $A$, which will produce a signal that encodes 2 . We would then connect $A$ to $B$, which will allow the signal to propagate between the two groups of neurons. However, as the signal passes between the two neuron groups, new decoding instructions are given to $B$, which in this example would be, "find the decoding weights that minimize the representational error of $f(x) . "$ Once this process occurs, if we transition from the neuron-space of $B$ to the state-space it represents, we will get an approximation of 4 , which is the desired result.

Speaking formally again, connections between neurons (synapses) are unidirectional, with the amount of current injected into downstream neurons being dependent on a synaptic weight matrix that describes the strength of the connections between neurons; this weight matrix serves as the neuron response function in eq. 1, and is computed as the product of the decoding weights for the transformation in the first group of neurons, the encoding weights of the downstream neurons, and the transformation itself (Bekolay et al., 2014). Similarly, decoding transformations is the same as decoding the original representation, only the original decoders from eq. 2 are replaced with decoders for the transformation (Eliasmith, 2013, p. 398).

\subsection{NEF Dynamics ${ }^{3}$}

Finally, dynamics draws on concepts from control theory, namely that the statespace representations of neuron groups are equivalent to the state-space variables of dynamical systems (Bekolay et al., 2014). In this regard, a recurrent connection between

\footnotetext{
3 Parts of this section have been taken from Riley and Davies (Forthcoming) with the authors' permission.
} 
a neuron group can be used to model the non-static nature of cognitive processes by treating the neuron group as an integrator (Eliasmith, 2013, p. 58-60). That said, these cognitive processes are rarely a perfect system, thus dynamics allow representations to degrade over time according to the postsynaptic current(s) of the neuron(s) (Eliasmith, 2013, p. 58-60). 


\section{Chapter: Overview of Model}

Once again, the goal of this research is to build a biologically plausible neuron model that can replicate the rotation aspect of the Shepard and Metzler (1971) paradigm. To do this, I built a series of neuron groups (referred to as "ensembles" in Nengo), and connected them together in such a way that they function to (i) perform a continuous, whole unit rotation, and (ii) determine when the object is brought into alignment with a second object. These objects take the form of cube assemblies, and are represented in a polar coordinate system that is discretized according to visual acuity, which itself resides in an egocentric frame of reference. However, because of limited computing resources, there is a trade-off between the biological accuracy of the neurons and the biological accuracy of the spatial map. That is, the larger (smaller) the spatial map, the less (more) complex the neurons. As such, I ran multiple simulations of the model, varying the neuron type and size of the spatial maps as needed.

\subsection{Topology of the Network}

Figure 3 shows six ensembles of neurons [circles: working memory (WM), motor monitor $(\mathrm{MM})$, spatial representation $\left(\mathrm{SPP}_{\mathrm{R}}\right)$, spatial transformation $\left(\mathrm{SPP}_{\mathrm{T}}\right)$, associative memory (AM), and output (Out)], and three input nodes [squares: the spatial map the is to be rotated $\left(\mathbf{M}_{1}\right)$, motor input, and the spatial map that rotated maps are to be compared against $\left(\mathbf{M}_{2}\right)$ ] comprise the network, with one integrator $(\Psi)$ that instructs movement away from the canonical orientation, and two functions $\left[f\left(\mathbf{M}_{1}, \Psi\right)\right.$ and $\left.g\left(\mathbf{M}_{R}, \mathbf{M}_{2}\right)\right]$ that rotate $\mathbf{M}_{1}$ and compare $\mathbf{M}_{R}$ (the rotated version of $\mathbf{M}_{1}$ ) with $\mathbf{M}_{2}$, respectively (Figure 3). In both spatial maps, the presence/absence of an object at a location in space is denoted 
by $1 /-1$, with the two inputs nodes inserting a vectorization of their respective spatial map.

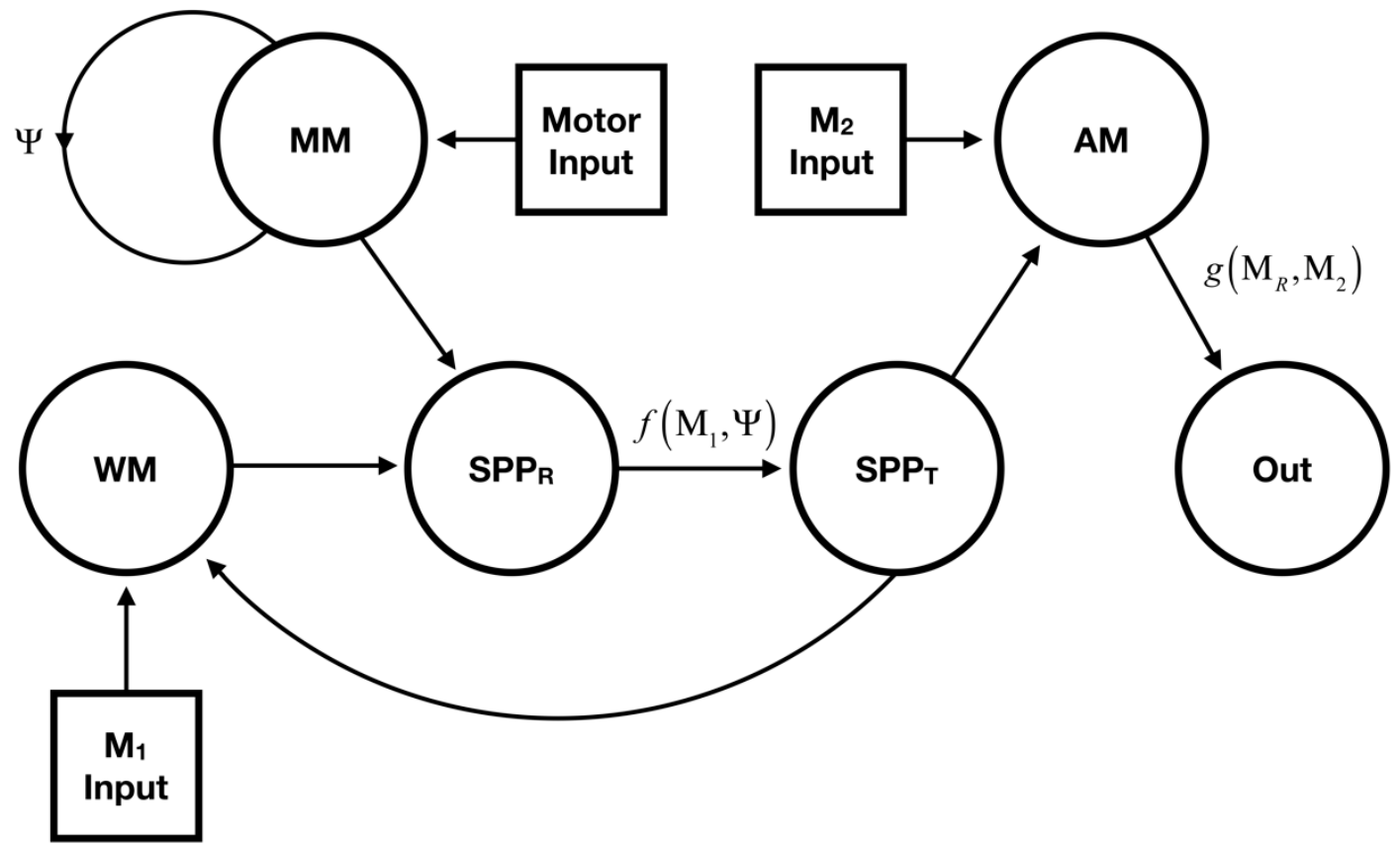

Figure 3: Network Topology

Squares are input nodes. Circles are neuron ensembles. Arrows specify the direction of connection. WM: working memory, $\mathbf{S P P}_{\mathbf{R}}$ : spatial representation, $\mathbf{S P P}_{\mathbf{T}}$ : spatial transformation, $\mathbf{M M}$ : motor monitor, AM: associative memory, out: output. $\mathbf{M}_{1}, \mathbf{M}_{2}$, and $\mathbf{M}_{R}$ are the spatial maps where $\mathbf{M}_{R}$ is the rotated version of $\mathbf{M}_{1} . \Psi$ is the integrator that instructions rotation. $\boldsymbol{f}\left(\mathbf{M}_{1}, \Psi\right)$ computes the rotation. $\boldsymbol{g}\left(\mathbf{M}_{R}, \mathbf{M}_{2}\right)$ compares the two spatial maps. 


\subsection{Parameters}

All neurons are either spiking-leaky-integrate-and-fire (sLIF) or non-spikingleaky-integrate-and-fire (LIF), depending on the simulation, and have encoding intercepts that are drawn from a uniform distribution between -1 and 1. Moreover, although ensembles and their connections have a wealth of additional parameters that can be adjusted, I only alter some, with the remaining being set to their default values or automatically computed [Nengo Source Code].

The WM ensemble takes as input $\mathbf{M}_{1}$ (from the WM node) and a rotated version of $\mathbf{M}_{1}$, denoted $\mathbf{M}_{R}$, from SPP $\mathrm{T}_{\mathrm{T}}$. Further, WM contains 10,000 neurons whose dimensionality - or how large a state-space it can represent - is equal to the total number of elements in $\mathbf{M}_{1}$ and $\mathbf{M}_{2}$, combined, with a radius - or the range of values the ensemble can represent - of 2 , thus allowing for representations between -2 and 2 .

The MM ensemble takes in the motor input, which is always 1 as I am doing continuous, clockwise rotations, as well as the output from the integrator, $\Psi$. Further, MM contains 1,500 neurons whose dimensionality is 1 , with a radius equal to the amount of time the model is simulating (e.g. 6 seconds). Finally, the synaptic time-constant of MM's recurrent connection was set to one second, which, in theory, will lead to new rotation instructions being generated every second, and thus produce a rotation rate of one unit per second.

The $\mathrm{SPP}_{\mathrm{R}}$ ensemble receives as input the canonical orientation of $\mathbf{M}_{1}$ from $\mathrm{WM}$, as well as rotation instructions from MM. It contains 10,000 neurons, with a dimensionality equal to the total number of elements in $\mathbf{M}_{1}$, plus one extra dimension for the rotation instructions. Moreover, the radius is equal to the amount of time the model is 
simulating, plus one. Finally, the connections between $\mathrm{WM} / \mathrm{MM}$ and $\mathrm{SPP}_{\mathrm{R}}$ are direct, which means $\mathrm{SPP}_{\mathrm{R}}$ forms a perfect representation of the signal it receives from WM/MM (that is, there is no representational drift when moving from WM/MM to $\mathrm{SPP}_{\mathrm{R}}$, though, as expected, there is representational drift/error throughout other parts of the model). This was done because connection weights across direct connections get computed at each time-step, as opposed to once during the build phase [Nengo Source Code]. This allows $f\left(\mathbf{M}_{1}, \Psi\right)$ to be computed at each time-step.

The $\mathrm{SPP}_{\mathrm{T}}$ ensemble receives input from $\mathrm{SPP}_{\mathrm{R}}$, and forms a representation of $\mathbf{M}_{R}$ via the computation of $f\left(\mathbf{M}_{1}, \Psi\right) . \mathrm{SPP}_{\mathrm{T}}$ is also comprised of 10,000 neurons, has a dimensionality equal to the number of elements in $\mathbf{M}_{R}$, and a radius of 2 .

$\mathrm{AM}$ has all of the same parameters as $\mathrm{WM}$, only it receives input from $\mathrm{SPP}_{\mathrm{T}}$, and also has a direct connection so $g\left(\mathbf{M}_{R}, \mathbf{M}_{2}\right)$ can be computed at each time-step.

Finally, the output ensemble has 1,500 neurons, receives input from AM, has a dimensionality of 2 (one dimension to specify if $\mathbf{M}_{R}=\mathbf{M}_{2}$, and one dimension for the cosine similarity between $\mathbf{M}_{R}$ and $\mathbf{M}_{2}$, which, theoretically, gives us an approximation of how far away $\mathbf{M}_{R}$ is from $\mathbf{M}_{2}$ ), as well as a radius of 2 . 


\section{Chapter: Method}

Overall, the model was used in three sets of simulations: (i) two-dimensional rotation focusing on the biological accuracy of neurons, (ii) three-dimensional rotation focusing on the biological accuracy of spatial maps, and (iii) three-dimensional rotation focusing on the biological accuracy of neurons. Each set simulated pairs of spatial maps at various angular discrepancies. Further, I will evaluate each set of simulations according to the following criteria: (i) they can successfully rotate $\mathbf{M}_{1}$ into alignment with $\mathbf{M}_{2}$, and can do so at various degrees of angular separation; (ii) they produce linear RTs (how long it takes the model to bring $\mathbf{M}_{1}$ into alignment with $\mathbf{M}_{2}$ ). All threedimensional rotations are clockwise about the z-axis. All two-dimensional rotations are clockwise.

\subsection{Two-Dimensional Rotation: Neuron-Focused}

To perform two-dimensional rotation, the entire three-dimensional map was constructed, but only locations within the top depth slice had objects present (that is, depth sections two and three were comprised entirely of empty space). Moreover, $\mathbf{M}_{1}$ was constructed as

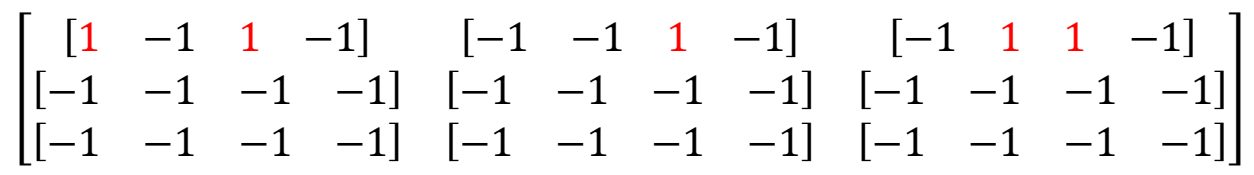

and $\mathbf{M}_{2}$ as

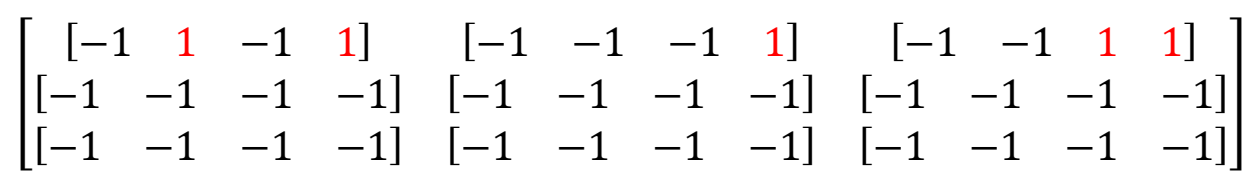

The primary focus of this thesis is three-dimensional rotation, so as a proof of concept, only one, two-dimensional simulation was run. 


\subsection{Three-Dimensional Rotation: Map-Focused}

Here, simulations used LIF neurons and $3 \times 3$ spatial maps discretized into 8 sections per circle, for a total of 72 voxels. $\mathbf{M}_{1}$ was constructed as

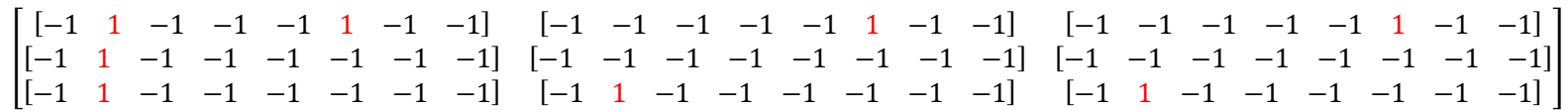
with a corresponding $\mathbf{M}_{2}$ at $1-7$ unit rotations. Thus, seven map-focused simulations were run.

\subsection{Three-Dimensional Rotation: Neuron-Focused}

In contrast to map-focused simulations, neuron-focused simulations used sLIF neurons and $3 \times 3$ spatial maps discretized into 4 sections per circle, for a total of 36 voxels. $\mathbf{M}_{1}$ was constructed as

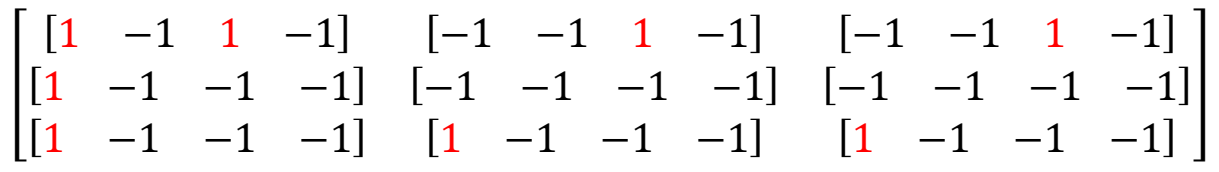

though the smaller maps means a corresponding $\mathbf{M}_{2}$ at 1, 2, and 3 unit rotations. Thus, three neuron-focused simulations were run.

\subsection{Rotation Function}

To perform rotations, $f\left(\mathbf{M}_{1}, \Psi\right)$ was computed across $\mathrm{SPP}_{\mathrm{R}} \rightarrow \mathrm{SPP}_{\mathrm{T}}$ at every time-step. At a computational level, $f\left(\mathbf{M}_{1}, \Psi\right)$ works according to Algorithm 1 below, which, every second, rotates the canonical spatial map $\left(\mathbf{M}_{1}\right)$ by $\Psi$ units ( $\Psi$ increments its value by 1 every second via MM). 
Algorithm 1: Rotate Spatial Map

Input: a vector containing $\mathbf{M}_{1}$ : canonical spatial map, $\Psi$ : rotation instruction

Output: a vector containing $\mathbf{M}_{R}$ : $\mathbf{M}_{1}$ rotated according to $\Psi$

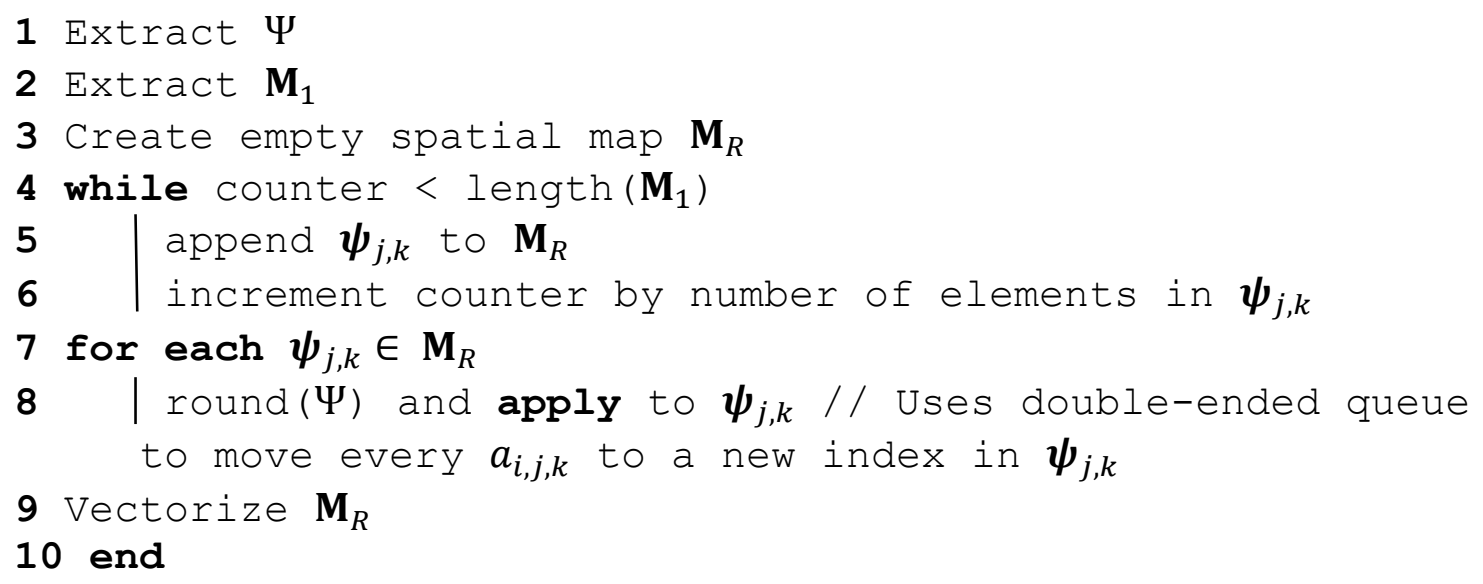

At a conceptual level, $f\left(\mathbf{M}_{1}, \Psi\right)$ works by rotating every point within the spatial map one unit per second along the intended axis. Although this runs counter to our intuitions, a deeper dive reveals that it makes sense as the approach does not require isolating points from empty space (see below). Moreover, in multi-object scenes, my thinking is that attending to a specific object isolates the object within $\mathbf{M}$ from $\mathbf{M}^{+}$; the object may be placed within the larger scene during rendering in the visual buffer, but when an object is selected for the purpose of rotation, it is separated from other aspects of the scene. That said, I also suspect the SPP can perform multiple transformations in parallel, thus allowing a person to imagine two objects side-by-side, but rotating in opposite directions. Finally, in cases where the stimuli demands a piecewise rotation, the same process is used, only instead of building $\mathbf{M}$ from the entire stimuli, it is built from 
part of the stimuli, with the entire stimuli being treated as a multi-object scene residing in $\mathbf{M}^{+}$.

More importantly, separating points from empty space would, arguably, result in $\mathbf{M}_{1}$ being processed in such a way that it does not preserve space, and thus would not be depictive. That is, if we only rotate points, then what matters are the elements that surround each point in $\mathbf{M}_{1}$ (and its rotated version, $\mathbf{M}_{R}$ ); thus, arguably, adhering to the descriptive view of mental imagery. However, by rotating both points and empty space, the elements surrounding each point stay the same - though they may shift positions (e.g., move from left to right during a 180 degree rotation) - with $\mathbf{M}_{1}$ moving into a rotated position, $\mathbf{M}_{R}$, by virtue of existing within an egocentric frame of reference, thus adhering to the depictive view of mental imagery.

To put it another way, if we imagine each location in $\mathbf{M}_{1}$ as a chair, and the value at that location as a unique person in that chair, then only rotating points would be analogous to only having certain people switch chairs, which would mean that what matters in understanding the chair-person structure is who is sitting beside who (not what chair they are in), which is (arguably) descriptive as it does not depend on any sort of spatial layout. However, by rotating both points and empty space, which would be analogous to having every person move the same number of chairs over, then what matters in understanding the chair-person structure is what chair they are sitting in, which is depictive as it requires a spatial layout (where each chair is located, either in the context of the room, or a chair indexing system). That is, rotating points and empty space demands that the matrix structure is not coordinate free, as Pylyshyn (2003) suggests; but rather, is understood in terms of a coordinate system (polar) imposed by a frame of 
reference (egocentric). If we only rotated points, then the matrix structure could be understood in terms of a coordinate system, or independent of one. Finally - and I know I am harping on this, but it is an important point to drill home - to synthesize the concept into a single sentence: if we rotate points and empty space, a matrix structure can only form new representations if it is depictive; if we rotate points alone, a matrix structure can form new representations if it is depictive or descriptive.

When it comes to $\Psi$, it, again, operates such that it increments by 1 (roughly) every second, and when used in $f\left(\mathbf{M}_{1}, \Psi\right)$, it produces representations of a continuous rotation in $\mathrm{SPP}_{\mathrm{T}}$. As Pylyshyn (2003) correctly points out, it is easy to move from one location in a matrix to any other location, thus restrictions on movement must come from a source external to the matrix (p. 360-365). The MM and $\Psi$ act as this external source as $\Psi$ generated independent of $\mathbf{M}_{1}$, and is represented in a subspace of $S P P_{R}$ that is independent of $\mathbf{M}_{1}$. That said, there is no computational reason why $\Psi$ can not generate rotation instructions that end up producing representations of non-continuous rotations in $\mathrm{SPP}_{\mathrm{T}}$; however, on the whole, the MM and $\Psi$ appear to be highly constrained such that $f\left(\mathbf{M}_{1}, \Psi\right)$ produces representations of a continuous rotation (see Chapter 3).

\subsection{Comparison Function}

To check if $\mathbf{M}_{R}=\mathbf{M}_{2}, g\left(\mathbf{M}_{R}, \mathbf{M}_{2}\right)$ was computed across AM $\rightarrow$ Out at every time-step. At a computational level, $g\left(\mathbf{M}_{R}, \mathbf{M}_{2}\right)$ works according to Algorithm 2 below, which identifies what indexes in $\mathbf{M}_{2}$ and $\mathbf{M}_{R}$ contain points, then compares the two sets of indexes against each other. 
Algorithm 2: Compare Spatial Maps

Input: A vector containing $\mathbf{M}_{R}$ : the rotated version of $\mathbf{M}_{1}$, and $\mathbf{M}_{2}$ : the target spatial map

Output: A vector $\pi$ containing 1/-1: whether the two maps are in alignment, and a scalar between -1 and 1: the cosine similarity between $\mathbf{M}_{R}$ and $\mathbf{M}_{2}$

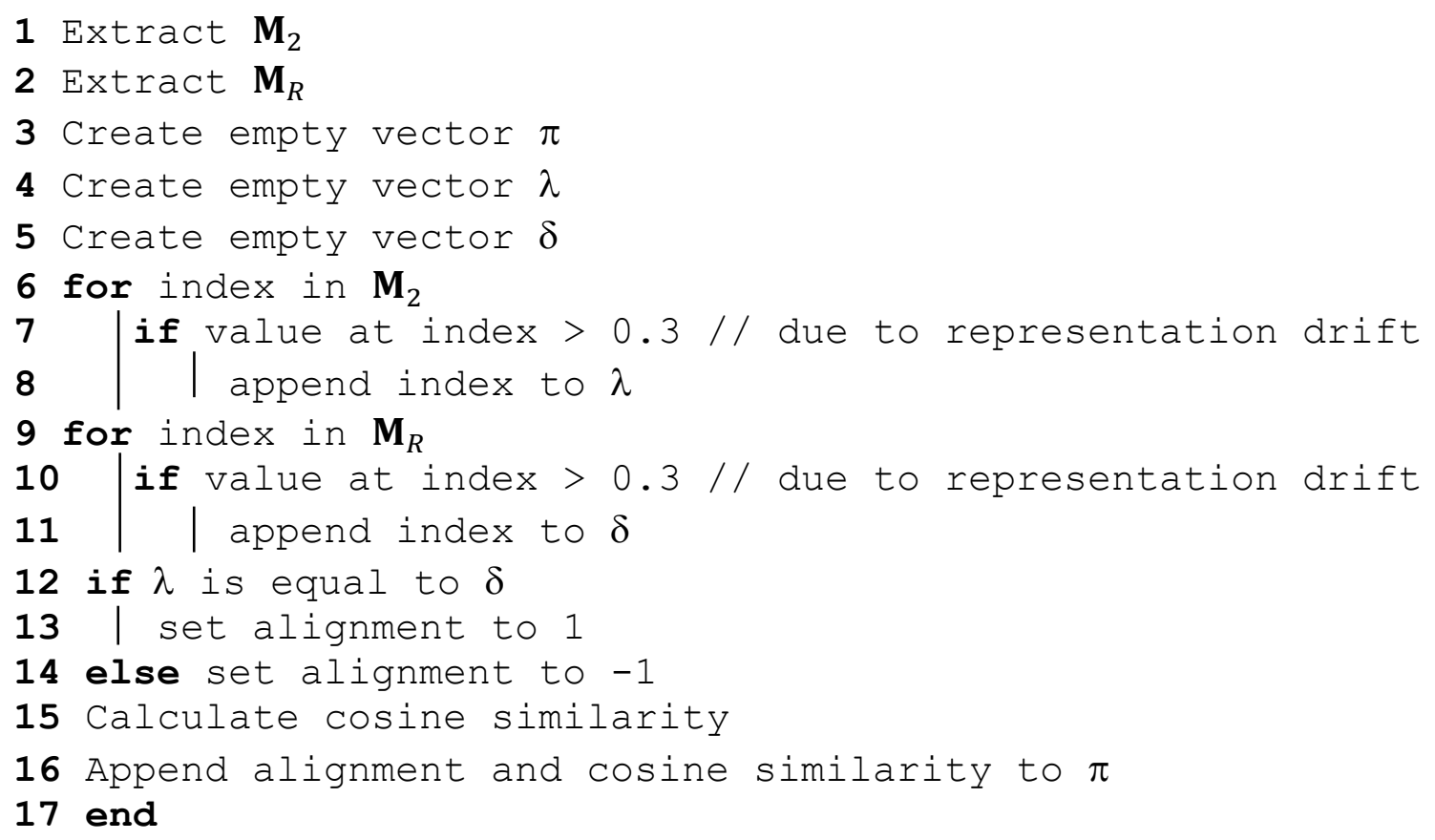

One could argue that if I am rotating both points and empty space, then I should check every index across both spatial maps. However, because representational drift is limited, the rotation of points entails the rotation of empty space, and depiction does not depend on the nature of comparison (as it does the nature of rotation), I contend it is viable to only check that the indexes of points match. 


\section{Chapter: Results}

\subsection{Two-Dimensional Rotation: Neuron-Focused}

As a proof of concept, a single, neuron-focused simulation of two-dimensional rotation was run. The model was simulated for six seconds. There was one unit of angular separation between maps. If the model is to behave exactly as planned, it should immediately rotate $\mathbf{M}_{1}$ into alignment with $\mathbf{M}_{2}$ (the model was not built to "linger" on the canonical orientation at the start of the simulation; it was built to immediately start rotating), hold $\mathbf{M}_{R}$ in alignment for 1 second, then perform 3 rotations out of alignment, then rotate back into alignment for 1 second, then out of alignment for 1 second.

Figure 4 shows data from a probe of the output ensemble. Interestingly, the sLIF neurons behaved in such a way that, despite having a synaptic time constant of 1 second across the $\mathrm{MM} \rightarrow \mathrm{MM}$ connection (Figure 5), the first iteration of $\mathbf{M}_{R}$ as only held for 0.5 seconds, with subsequent iterations of $\mathbf{M}_{R}$ being held for the expected 1 second. When we take this into account, we see that the model otherwise behaved as intended: Tracking the blue spike trains, it immediately rotated into alignment with $\mathbf{M}_{2}$, held that alignment for 0.5 seconds, then performed 3 more rotations that were out of alignment (holding each one for 1 second), then rotated back into alignment at the 3.5 second mark, held that alignment for 1 second, then performed two more rotations that were out of alignment (the additional rotation occurred because the first rotation was only held for 0.5 seconds, leaving an additional 0.5 seconds to perform and hold a rotation). This behaviour is supported by the cosine similarity, which immediately jumped to 1 , then fell down around 0.5 , spiking back up to 1 at the 3.5 second mark, then back down around 0.5 . 


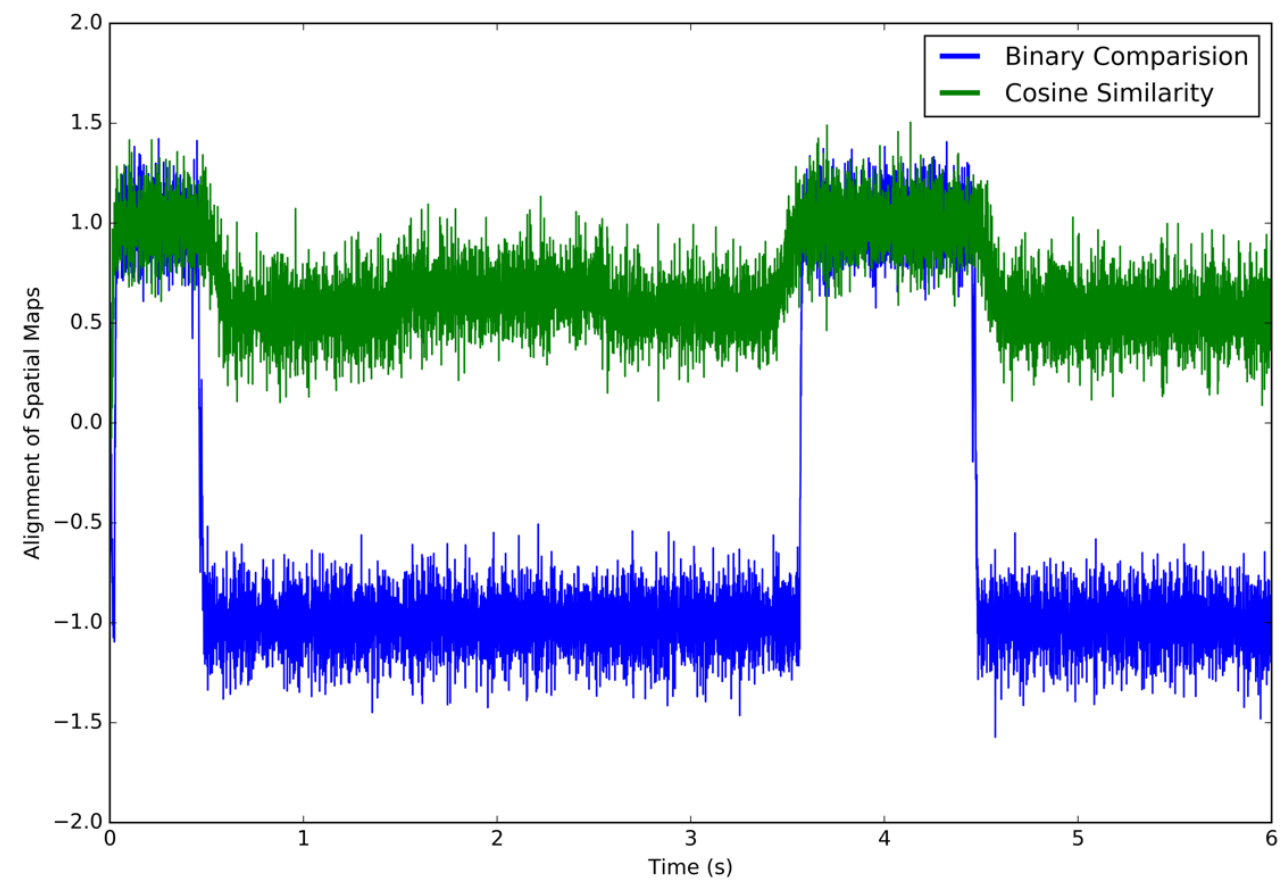

\section{Figure 4: Simulation 1: Output Ensemble}

Y-axis is the alignment of $\mathbf{M}_{R}$ with $\mathbf{M}_{2}$. X-axis is time in seconds. Blue is a binary comparison (1 they match; -1 they do not match). Green is the cosine similarity. Here, the model immediately rotated $\mathbf{M}_{\mathbf{1}}$ such that $\mathbf{M}_{R}$ was in alignment with $\mathbf{M}_{2}$, held that alignment for 0.5 seconds, then passed through a series of orientations that were not in alignment with $\mathbf{M}_{2}(0.5 \mathrm{~s}-3.5 \mathrm{~s})$, then once again rotated the map into alignment and held it for 1 second $(3.5 \mathrm{~s}-4.5 \mathrm{~s})$, and finally passed through orientations that were not in alignment $(4.5 \mathrm{~s}-6 \mathrm{~s})$. The main takeaways from this figure: (i) the model can successfully perform twodimensional rotations and identify when two spatial maps are in alignment, (ii) the initial rotation was only held for 0.5 seconds, not the expected 1 second. 


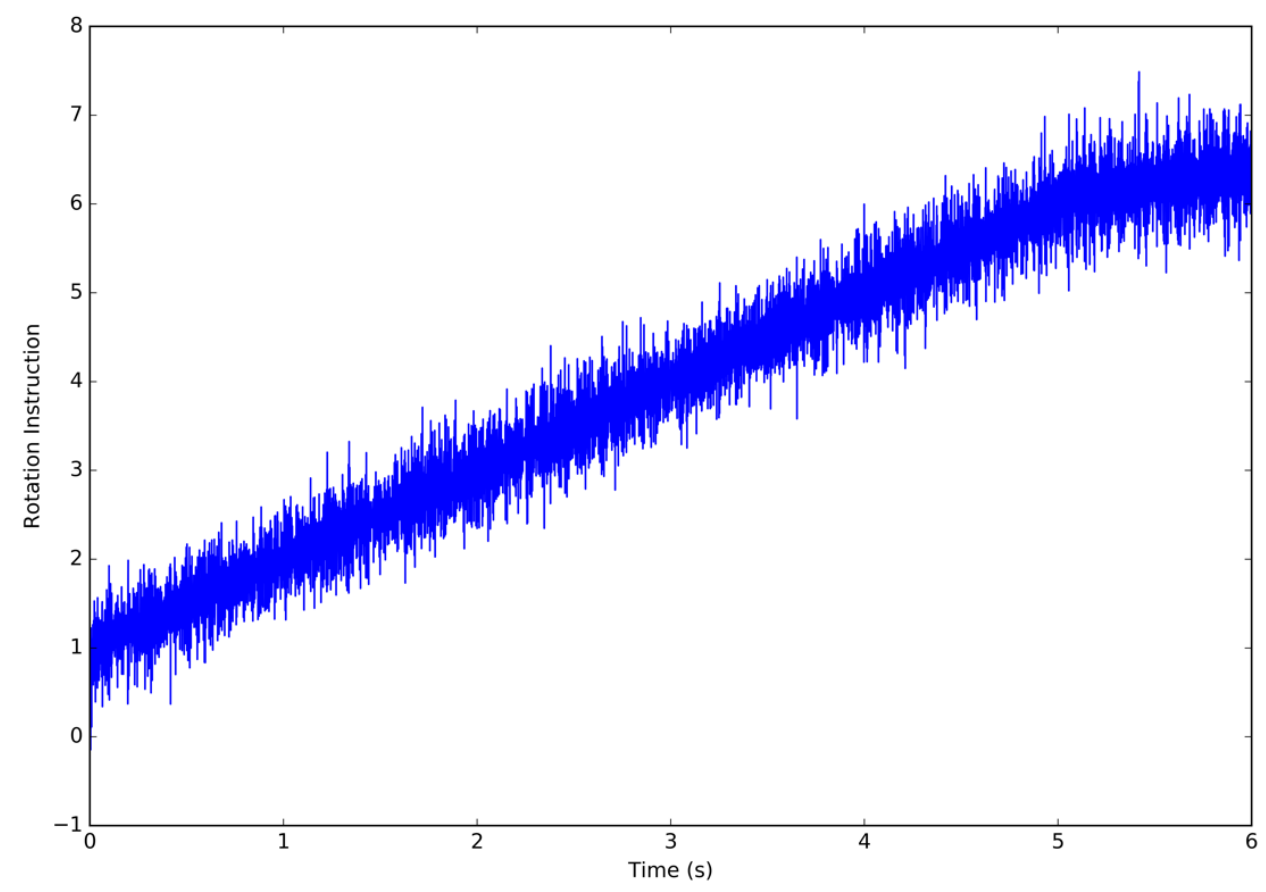

\section{Figure 5: Simulation 1: Motor Monitor Ensemble}

$\mathrm{Y}$-axis is the rotation instruction. $\mathrm{X}$-axis is time in seconds. The blue spike train is $\Psi$. Looking at the $\mathrm{y}$ intercept, we can see that the model immediately instructs a rotation of the spatial map, as intended. Moreover, around the 0.5 second mark, we can see that the value of $\Psi$ is approximately 1.5 , which explains why the model only held the initial rotation for 0.5 seconds as we round $\Psi$ to the nearest integer during $\boldsymbol{f}\left(\mathbf{M}_{1}, \Psi\right)$. Finally, $\Psi$ exhibits a clear, linear increase over time, which tells us that the synaptic time constant (1s) behaved as expected. 
To address the representation issues during the first second of the simulation, $f\left(\mathbf{M}_{1}, \Psi\right)$ was altered to make .8 the rounding cut-off instead of the conventional .5 , and the model run again (Figure 6).

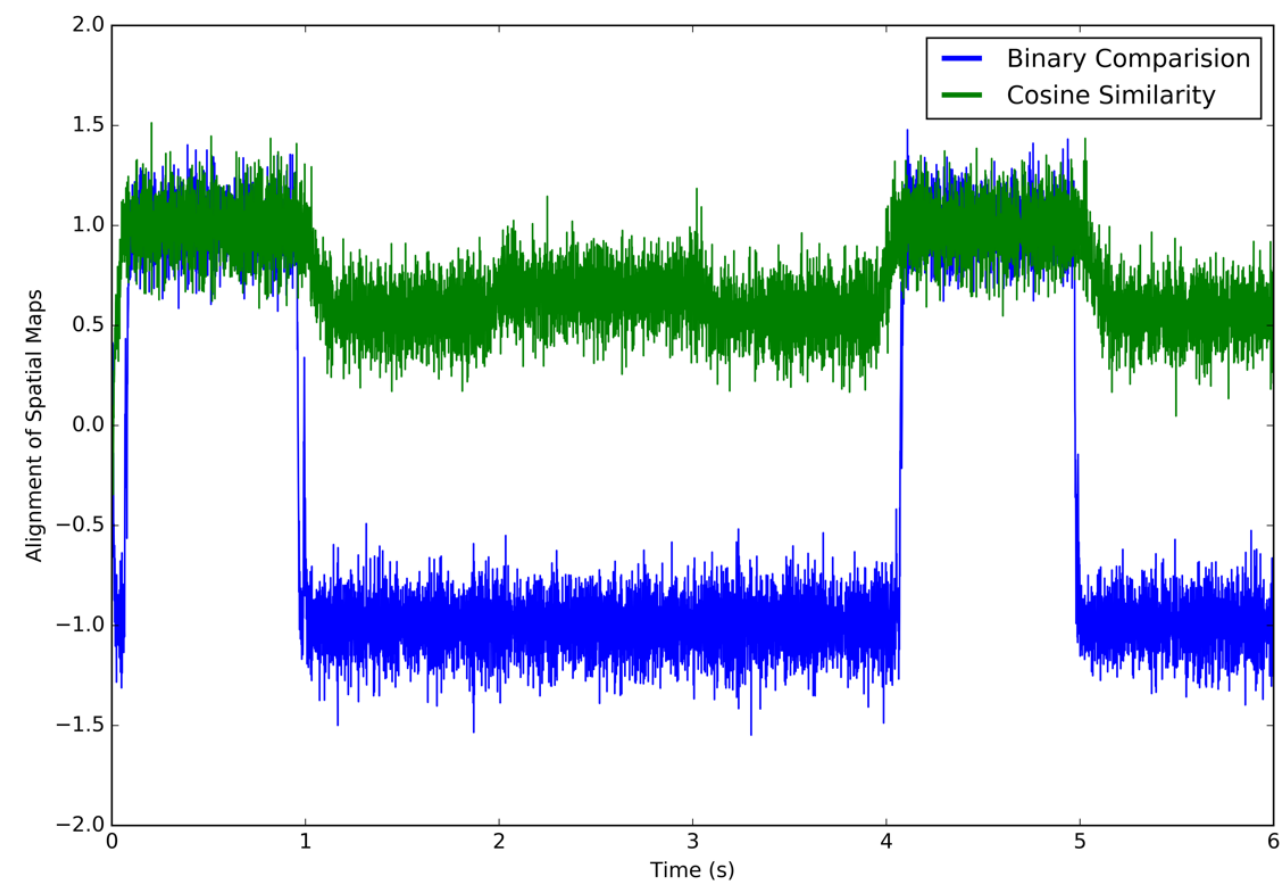

Figure 6: Simulation 2: Output Ensemble

$\mathrm{Y}$-axis is the alignment of $\mathbf{M}_{R}$ with $\mathbf{M}_{2}$. X-axis is time in seconds. Blue is a binary comparison (1 they match; -1 they do not match). Green is the cosine similarity. In contrast to Figure 4, we see that the model now holds the initial rotation for 1 second, and behaves exactly as expected across the entire 6 seconds. 


\subsection{Three-Dimensional Rotation: Map-Focused}

Next, seven map-focused simulations were run using LIF neurons and the updated rounding cut-off (.8). The first simulation had one unit of angular separation, with each subsequent simulation increasing the separation by one unit (Figures $7-15$ ). The model was always simulated for nine seconds to ensure we passed through every possible orientation. If the model is to behave as expected, we should see the first simulation immediately rotate $\mathbf{M}_{1}$ such that $\mathbf{M}_{R}$ aligns with $\mathbf{M}_{2}$, then rotate out of alignment for 7 seconds, then rotate back into alignment (Table 1). All subsequent simulations should see the model rotate into alignment at the $1 \mathrm{~s}-7 \mathrm{~s}$ marks, respectively, with all other timesteps being out of alignment. Finally, because we are utilizing LIF neurons, our model should not exhibit the spiking behaviour seen during two-dimensional simulations.

Table 1: Expected vs. Actual Model Behaviour (Map-Focused)

\begin{tabular}{lrr} 
Units of Angular Separation & Expected Time-Step of Alignment & Actual Time-Step of Alignment \\
\hline 1 & $0 \mathrm{~s}-1 \mathrm{~s} \& 8 \mathrm{~s}-9 \mathrm{~s}$ & $0 \mathrm{~s}-1 \mathrm{~s} \& 8 \mathrm{~s}-9 \mathrm{~s}$ \\
2 & $1 \mathrm{~s}-2 \mathrm{~s}$ & $1 \mathrm{~s}-2 \mathrm{~s}$ \\
3 & $2 \mathrm{~s}-3 \mathrm{~s}$ & $2 \mathrm{~s}-3 \mathrm{~s}$ \\
4 & $3 \mathrm{~s}-4 \mathrm{~s}$ & $3 \mathrm{~s}-4 \mathrm{~s}$ \\
5 & $4 \mathrm{~s}-5 \mathrm{~s}$ & $4 \mathrm{~s}-5 \mathrm{~s}$ \\
6 & $5 \mathrm{~s}-6 \mathrm{~s}$ & $5 \mathrm{~s}-6 \mathrm{~s}$ \\
7 & $6 \mathrm{~s}-7 \mathrm{~s}$ & $6 \mathrm{~s}-7 \mathrm{~s}$
\end{tabular}

Note: $\mathbf{M}_{2}$ never takes the same form as the canonical orientation 


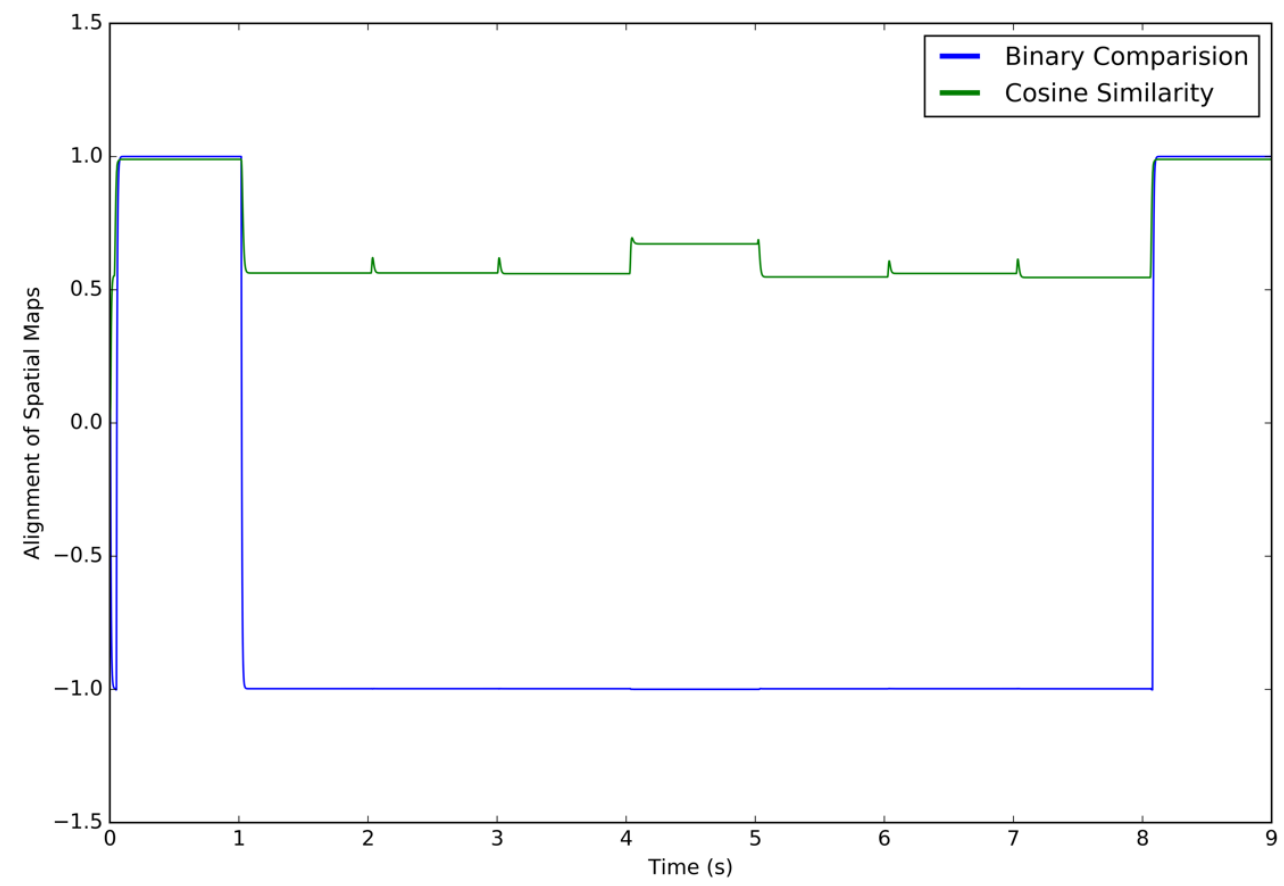

Figure 7: Simulation 3: Output Ensemble: 1 unit of separation

Y-axis is the alignment of $\mathbf{M}_{\boldsymbol{R}}$ with $\mathbf{M}_{\mathbf{2}}$. X-axis is time in seconds. Blue is a binary comparison (1 they match; -1 they do not match). Green is the cosine similarity. Here, the model correctly rotates into alignment with $\mathbf{M}_{2}$ at the start of the simulation and at the 8 second mark, with seven out of alignment rotations occurring at $1 \mathrm{~s}$ through $7 \mathrm{~s}$. Finally, the fact we rotated back into alignment at $8 \mathrm{~s}$ helps confirm that we passed through every possible orientation during the simulation. 


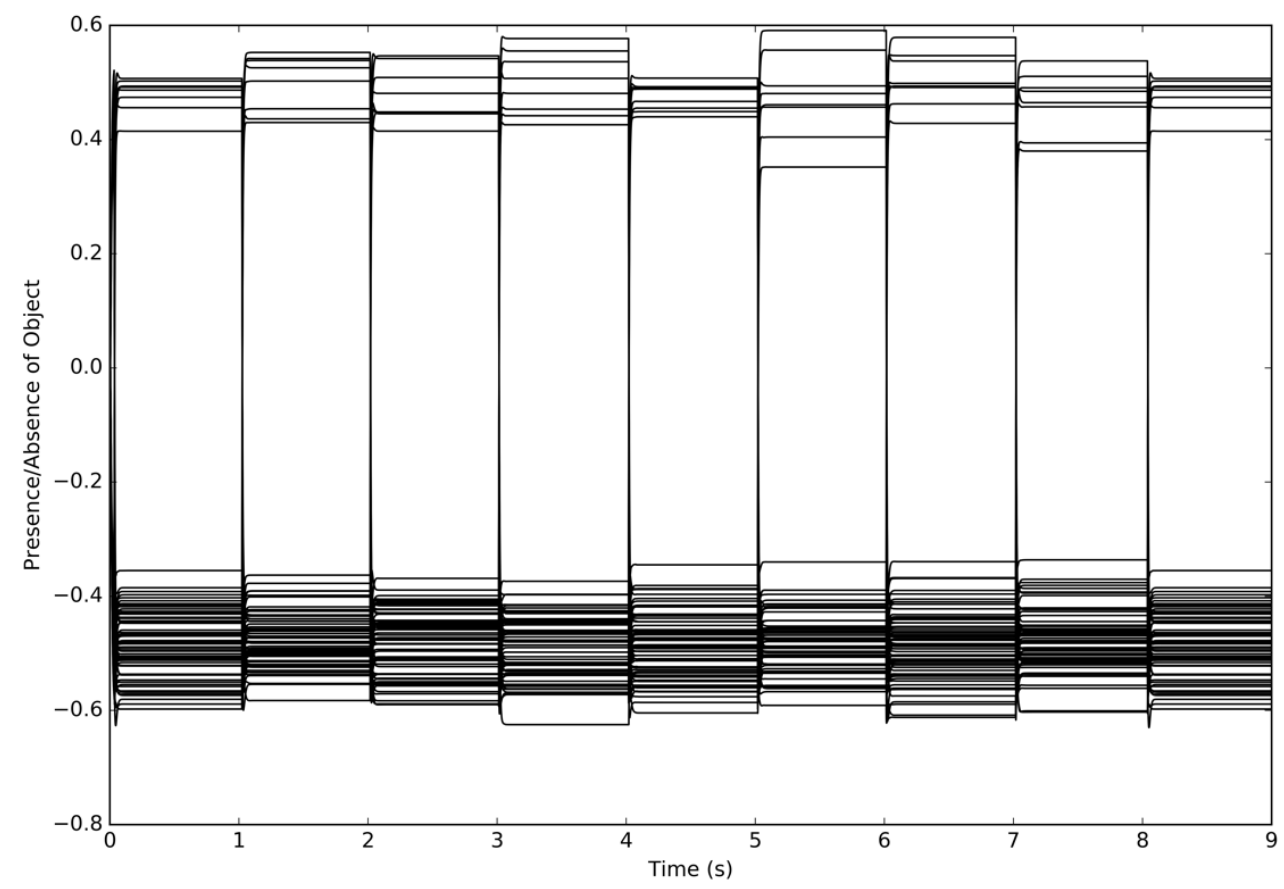

\section{Figure 8: Simulation 3: SPP Transform Ensemble: 1 unit of separation}

Here, every index in $\mathbf{M}_{\boldsymbol{R}}$ is represented as a unique line. $\mathrm{Y}$-axis is the presence/absence of a point at a location in space. $\mathrm{X}$-axis is time in seconds. Scanning across the graph, we can see a vertical line at every 1 second interval. This line is an overlay of all the indexes in $\mathbf{M}_{\boldsymbol{R}}$ whose values shift in response to a point moving into (or out of) that index. This provides further confirmation that the model is correctly performing rotations at a rate of one per second. Moreover, although there is some representational drift (y-axis values not being exactly $1 /-1$ at each index in $\mathbf{M}_{R}$ ), there is still a clear demarcation between presence/absence values. Finally, if we scan vertically and compare the y-values for each index between 0 s $-1 \mathrm{~s}$ and $8 \mathrm{~s}-9 \mathrm{~s}$, we can see that the two sets of $\boldsymbol{a}_{\boldsymbol{i}, j, \boldsymbol{k}}$ values are identical, which is what we would expect as those are the only two time-steps where an orientation is repeated. This analysis helps confirm that we passed through all possible orientations during the simulation, and is a handy, albeit unintended, byproduct of the model's representational drift (Note: this analysis would not be possible with perfect $1 /-1$ representations). 


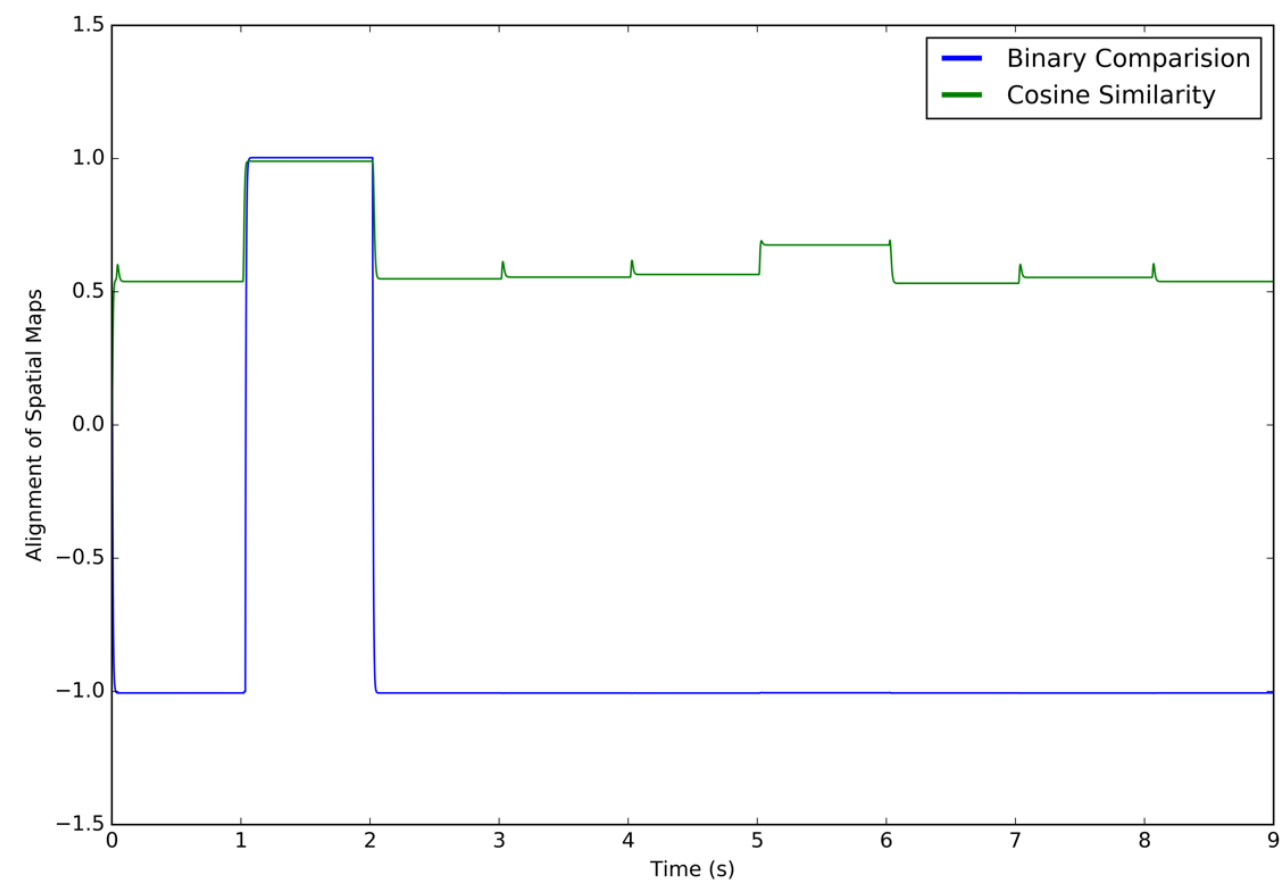

Figure 9: Simulation 4: Output Ensemble: 2 units of separation

Y-axis is the alignment of $\mathbf{M}_{R}$ with $\mathbf{M}_{2}$. X-axis is time in seconds. Blue is a binary comparison (1 they match; -1 they do not match). Green is the cosine similarity. In contrast to Figure 7 (one unit of separation), we see here that the model correctly rotates into alignment at the 1 second mark, with all remaining rotations being out of alignment. 


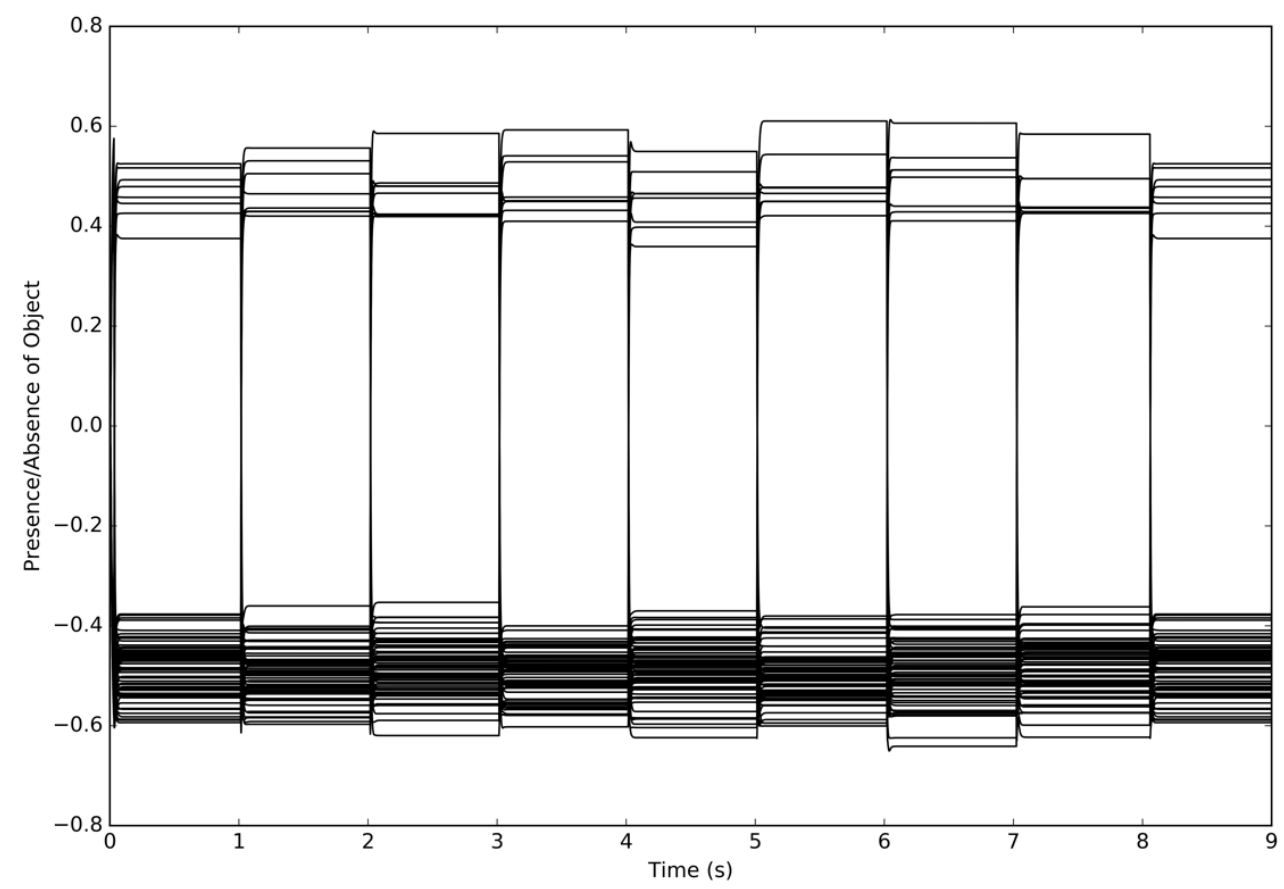

\section{Figure 10: Simulation 4: SPP Transform Ensemble: 2 units of separation}

Here, every location in $\mathbf{M}_{R}$ is represented as a unique line. $\mathrm{Y}$-axis is the presence/absence of a point at a location in space. $\mathrm{X}$-axis is time in seconds. As with Figure 8, we once again have confirmation that rotations are occurring once every second (vertical lines at 1 second intervals). Moreover, scanning vertically we see that the first and last rotation $(0 s-1 s$ and $8 s-9 s)$ share the same orientation, helping confirm that we passed through every possible orientation during the simulation. Finally, comparing this figure with Figure 8 reveals that the two are highly similar. This is to be expect as each simulation, regardless of angular separation, starts with a rotation away from the canonical orientation, continuing all the way past the canonical orientation and back to where it started (thus why the first and last time-steps produce identical orientations). That said, the y-values for each index vary slightly due to different random seed; however, they have the same underlying structure, and as such I will no longer display $\mathbf{S P P}_{\mathbf{T}}$ data for remaining simulations. 


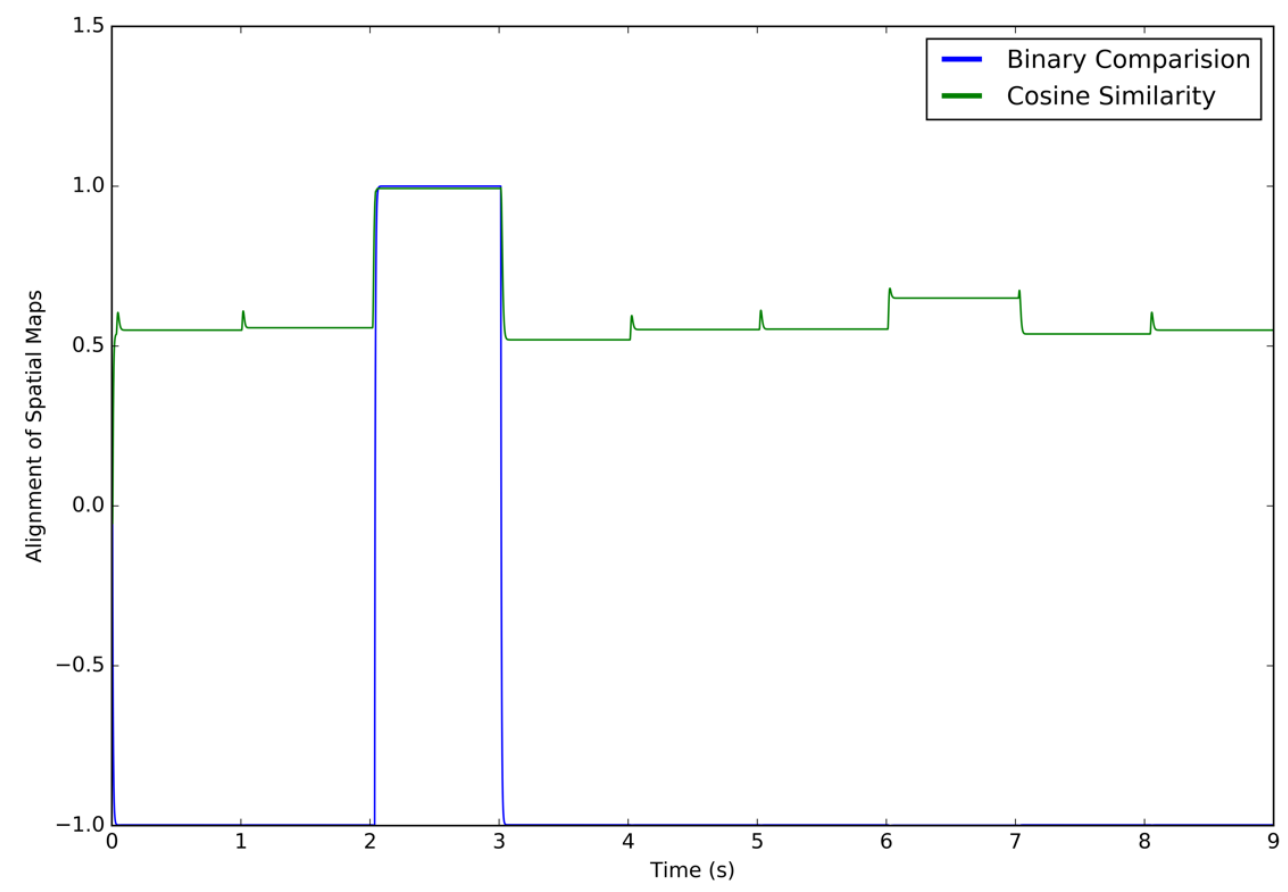

Figure 11: Simulation 5: Output Ensemble: 3 units of separation

Y-axis is the alignment of $\mathbf{M}_{R}$ with $\mathbf{M}_{2}$. X-axis is time in seconds. Blue is a binary comparison (1 they match; -1 they do not match). Green is the cosine similarity. The model once again rotates into alignment at the appropriate time-step (2s). 


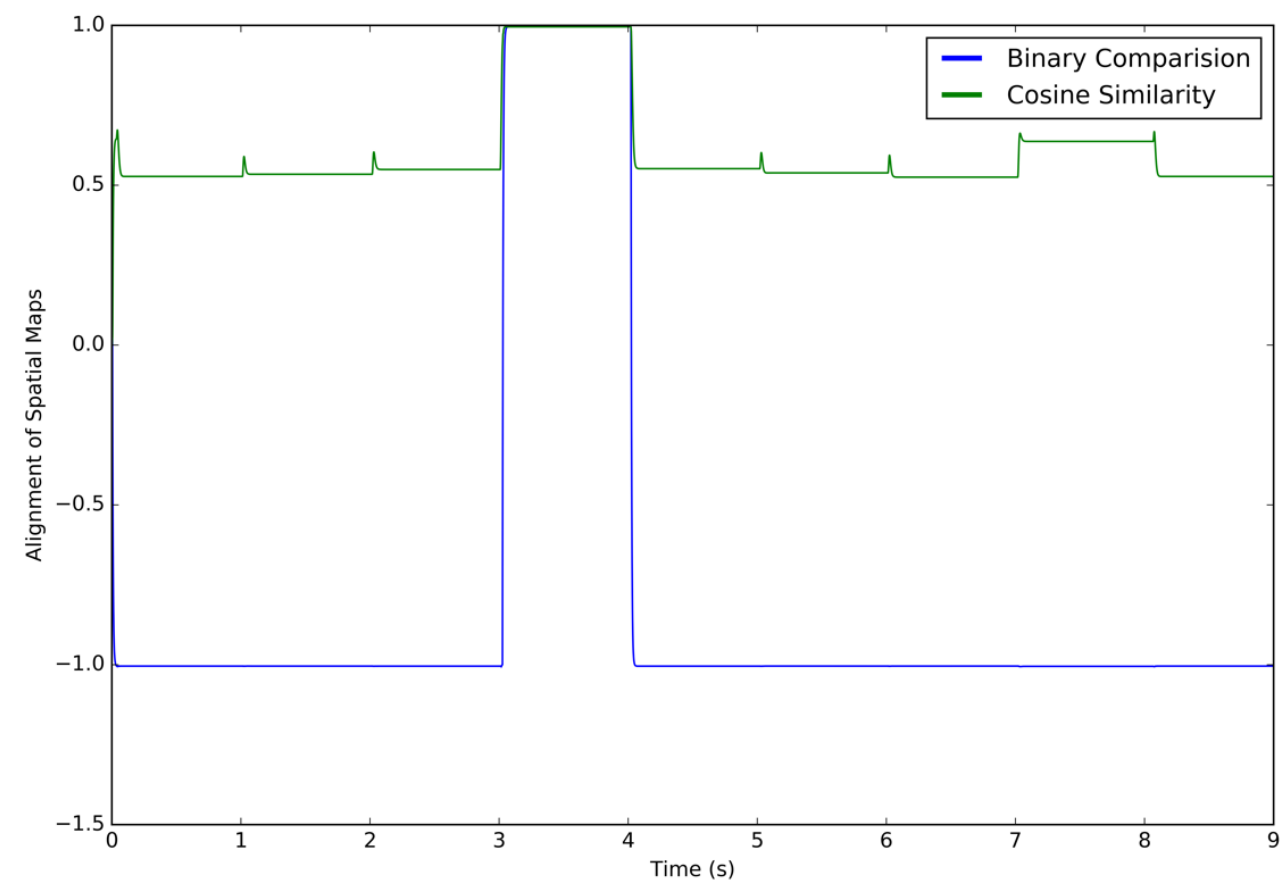

Figure 12: Simulation 6: Output Ensemble: 4 units of separation

Y-axis is the alignment of $\mathbf{M}_{R}$ with $\mathbf{M}_{2}$. X-axis is time in seconds. Blue is a binary comparison (1 they match; -1 they do not match). Green is the cosine similarity. The model once again rotates into alignment at the appropriate time-step (3s). 


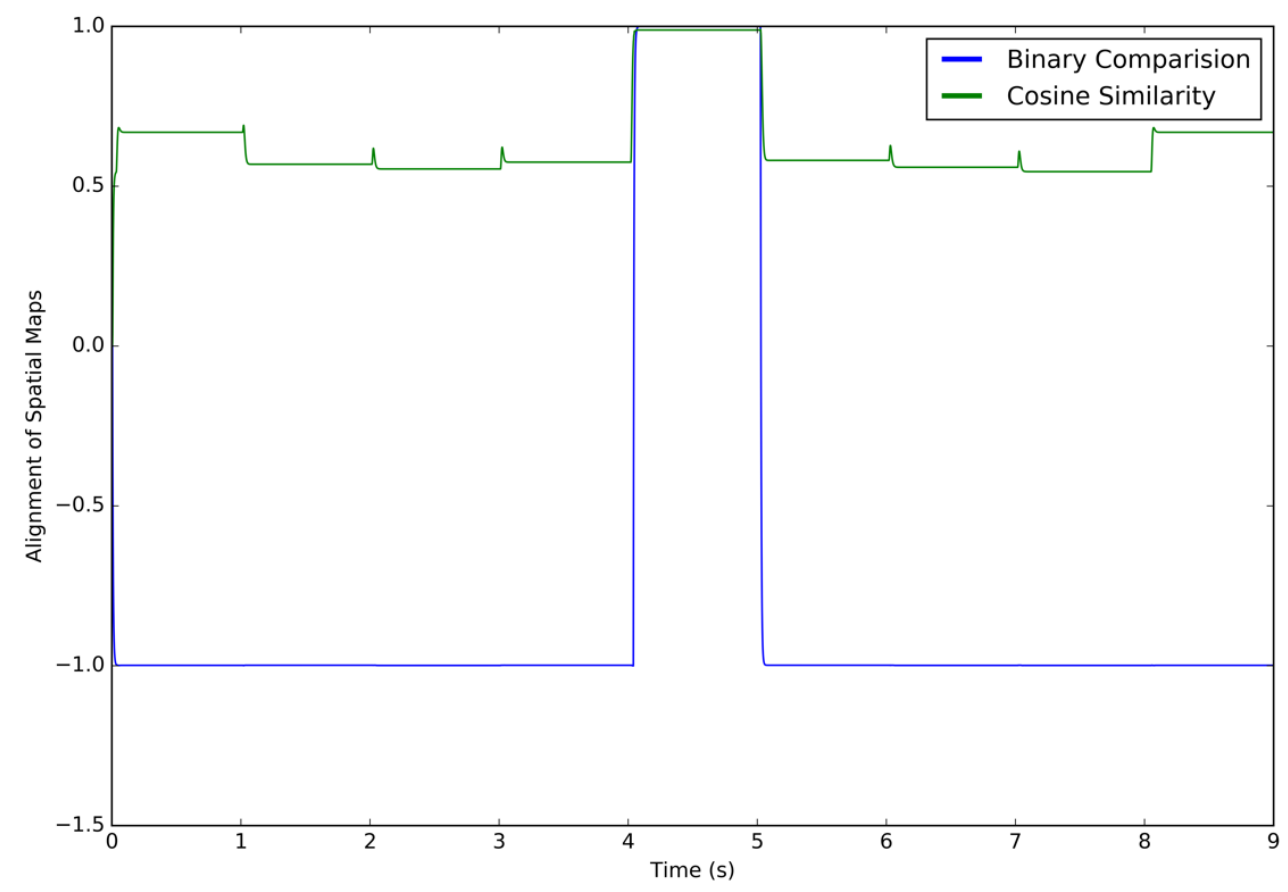

Figure 13: Simulation 7: Output Ensemble: 5 units of separation

$\mathrm{Y}$-axis is the alignment of $\mathbf{M}_{R}$ with $\mathbf{M}_{2}$. X-axis is time in seconds. Blue is a binary comparison (1 they match; -1 they do not match). Green is the cosine similarity. The model once again rotates into alignment at the appropriate time-step (5s). 


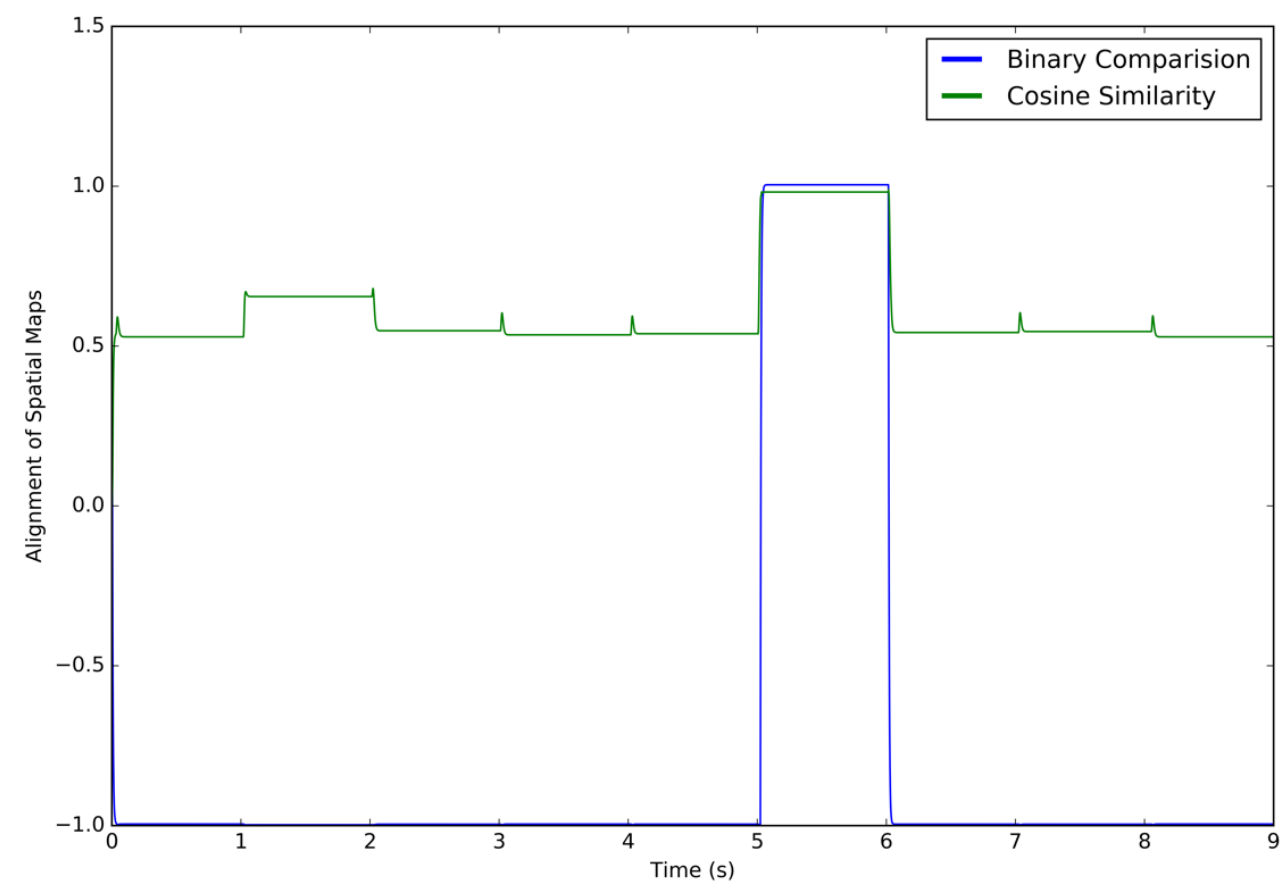

Figure 14: Simulation 8: Output Ensemble: 6 units of separation

$\mathrm{Y}$-axis is the alignment of $\mathbf{M}_{R}$ with $\mathbf{M}_{2}$. X-axis is time in seconds. Blue is a binary comparison (1 they match; -1 they do not match). Green is the cosine similarity. The model once again rotates into alignment at the appropriate time-step (6s). 


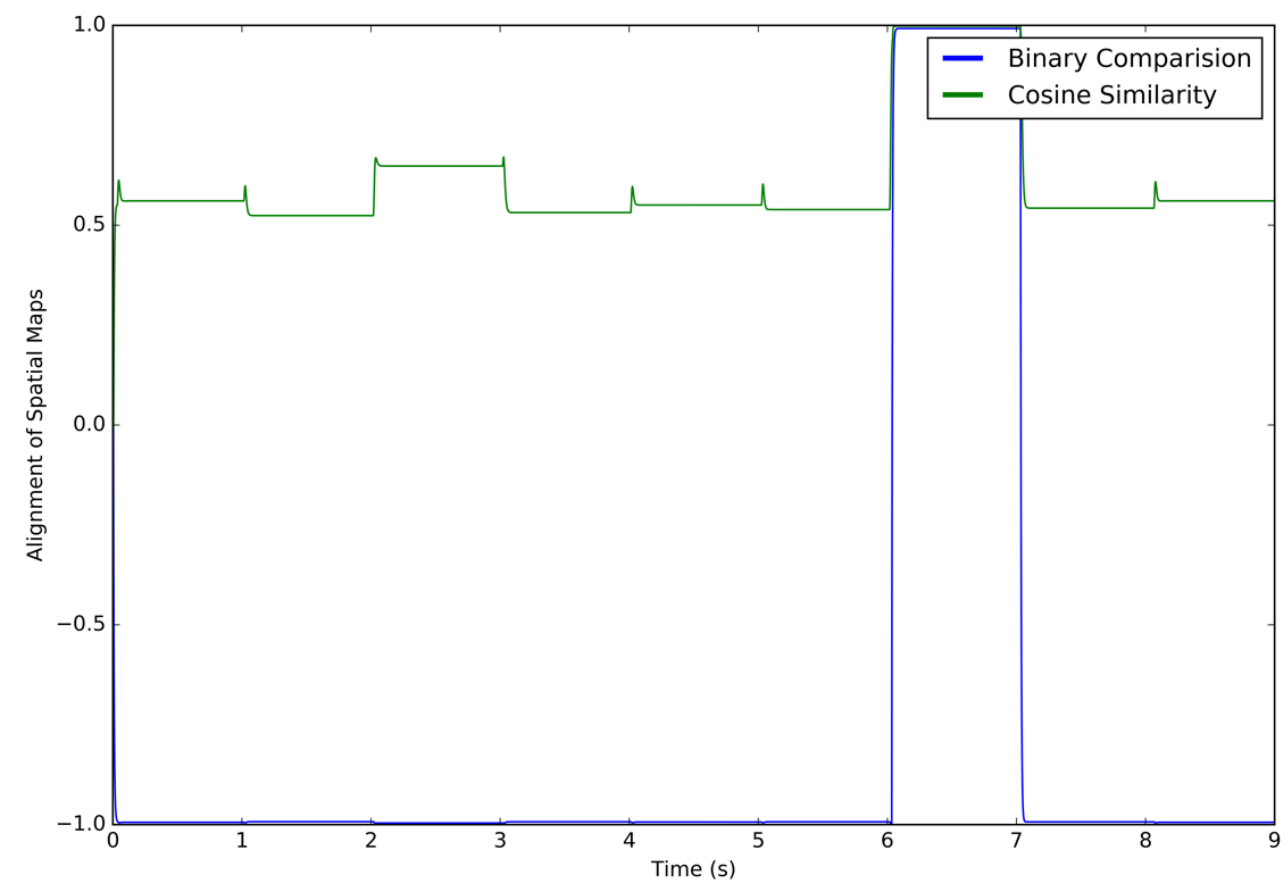

Figure 15: Simulation 9: Output Ensemble: 7 units of separation

Y-axis is the alignment of $\mathbf{M}_{\boldsymbol{R}}$ with $\mathbf{M}_{\mathbf{2}}$. X-axis is time in seconds. Blue is a binary comparison (1 they match; -1 they do not match). Green is the cosine similarity. The model once again rotates into alignment at the appropriate time-step (6s). 


\subsection{Three-Dimensional Rotation: Neuron-Focused}

Finally, three neuron-focused simulations were run using sLIF neurons and the updated rounding cut-off (.8). The first simulation had one unit of angular separation, with each subsequent simulation increasing the separation by one unit (Figures $16-\mathrm{xxx}$ ). The model was always simulated for five seconds to ensure we passed through every possible orientation. If the model is to behave as expected, we should see the first simulation immediately rotate $\mathbf{M}_{1}$ such that $\mathbf{M}_{R}$ aligns with $\mathbf{M}_{2}$, then rotate out of alignment for yyy seconds, then rotate back into alignment (Table 2). All subsequent simulations should see the model rotate into alignment at the $1 \mathrm{~s}-$ yys marks, respectively, with all other time-steps being out of alignment. Finally, because we are utilizing sLIF neurons, our model should exhibit the spiking behaviour seen during twodimensional simulations.

Table 2: Expected vs Actual Model Behaviour (Neuron-Focused)

\begin{tabular}{lrr} 
Units of Angular Separation & Expected Time-Step of Alignment & Actual Time-Step of Alignment \\
\hline 1 & $0 \mathrm{~s}-1 \mathrm{~s} \& 4 \mathrm{~s}-5 \mathrm{~s}$ & $0 \mathrm{~s}-1 \mathrm{~s} \& 4 \mathrm{~s}-5 \mathrm{~s}$ \\
2 & $1 \mathrm{~s}-2 \mathrm{~s}$ & $1 \mathrm{~s}-2 \mathrm{~s}$ \\
3 & $2 \mathrm{~s}-3 \mathrm{~s}$ & $2 \mathrm{~s}-3 \mathrm{~s}$
\end{tabular}

Note: $\mathbf{M}_{2}$ never takes the same form as the canonical orientation 


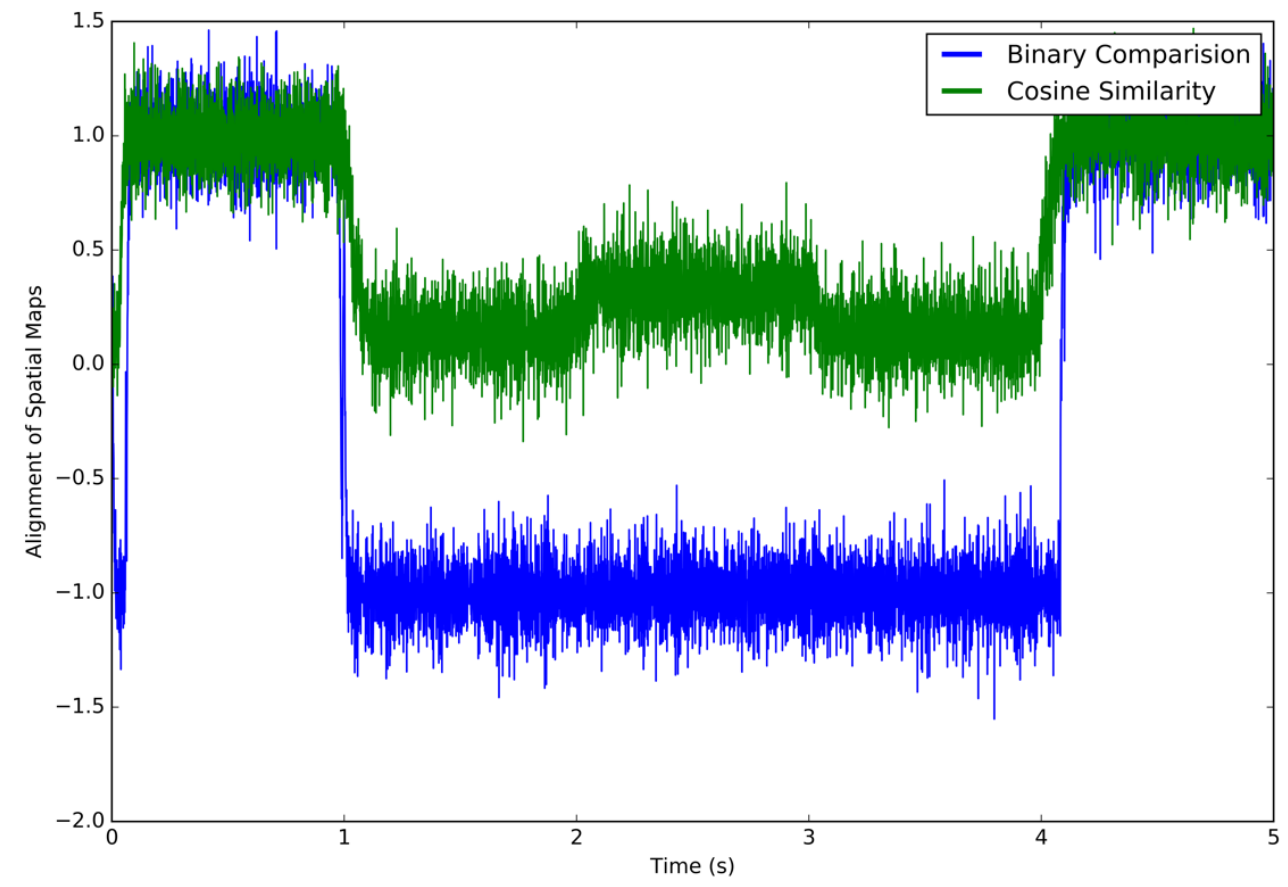

Figure 16: Simulation 10: Output Ensemble: 1 unit of separation

$\mathrm{Y}$-axis is the alignment of $\mathbf{M}_{R}$ with $\mathbf{M}_{2}$. X-axis is time in seconds. Blue is a binary comparison (1 they match; -1 they do not match). Green is the cosine similarity. The model correctly rotates into alignment at the appropriate intervals (first and last), with three out of alignment rotations occurring from $1 \mathrm{~s}-4 \mathrm{~s}$. 


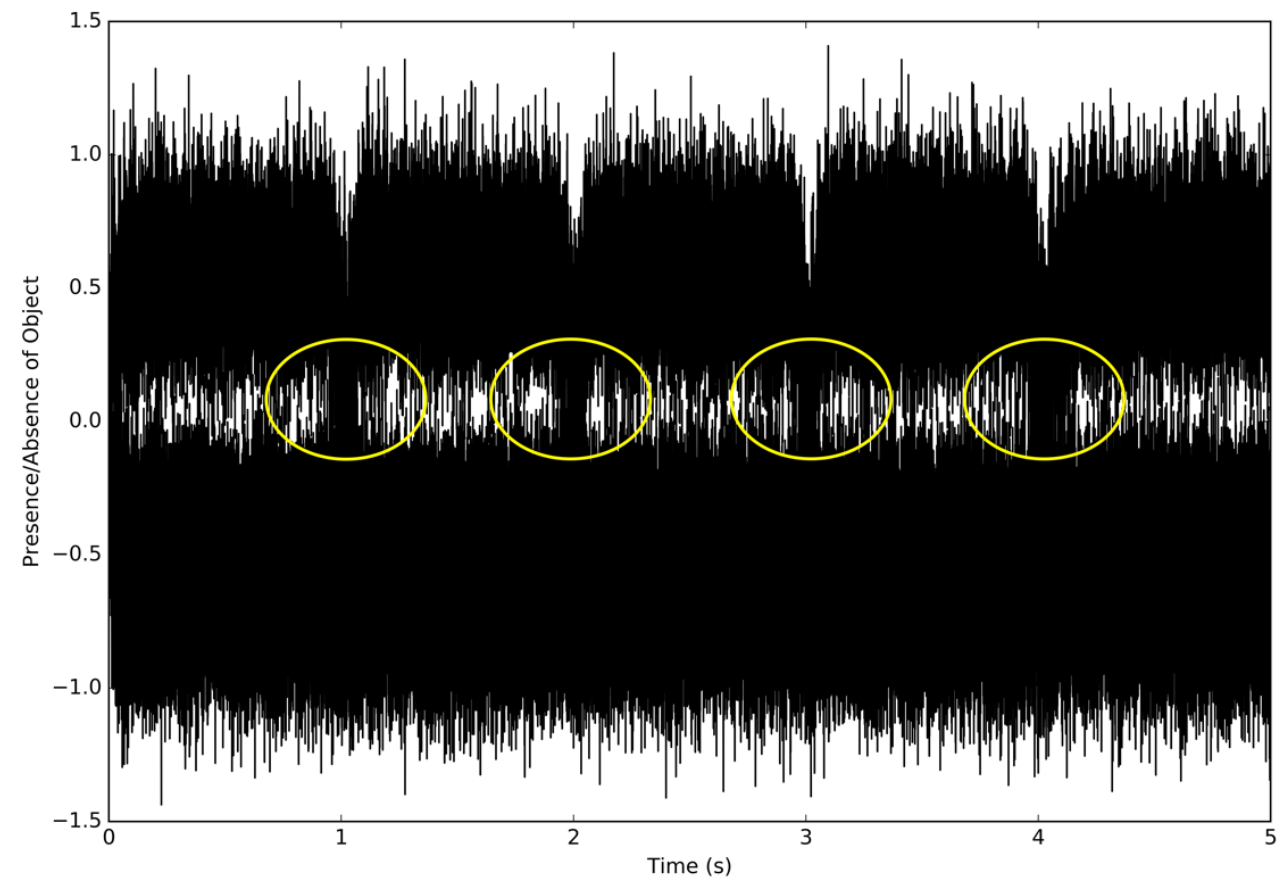

Figure 17: Simulation 10: SPP Transform Ensemble: 1 unit of separation

Here, every index in $\mathbf{M}_{\boldsymbol{R}}$ is represented as a unique line. $\mathrm{Y}$-axis is the presence/absence of a point at a location in space. $\mathrm{X}$-axis is time in seconds. Scanning across the graph, we can see a vertical line at every 1 second interval (yellow circles). This line is an overlay of all the indexes in $\mathbf{M}_{R}$ whose values shift in response to a point moving into (or out of) that index. This provides further confirmation that the model is correctly performing rotations at a rate of one per second. However, if we compare this figure with Figures $8 \& 10$ (SPP Transform under LIF neurons), we see that the neuron's spiking behaviour narrows the divide between the representations of the presence/absence of points in space; we can still delineate between the two [and can do so without altering $\boldsymbol{f}\left(\mathbf{M}_{1}, \Psi\right)$ or $\boldsymbol{g}\left(\mathbf{M}_{R}, \mathbf{M}_{2}\right)$ ], though any reduction in neurons - or increase in the size of spatial maps - will likely make the two indistinguishable as the model will not have enough neurons to generate a reasonably accurate encoding of the state-space. Given more resources, as we increase the number of sLIF neurons in the model, they will generate an increasingly accurate encoding of the state-space, which would make for a cleaner demarcation. 


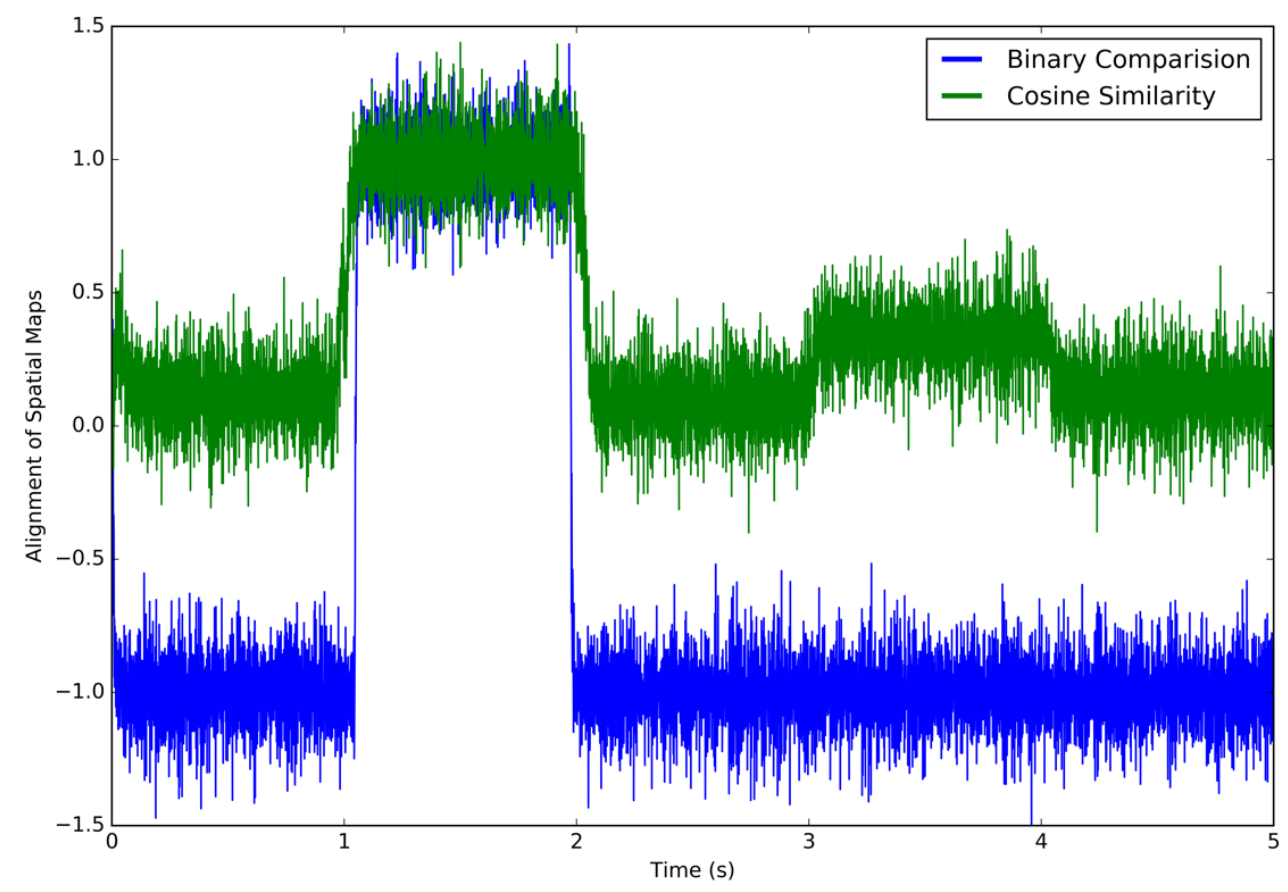

Figure 18: Simulation 11: Output Ensemble: 2 units of separation

$\mathrm{Y}$-axis is the alignment of $\mathbf{M}_{R}$ with $\mathbf{M}_{\mathbf{2}}$. X-axis is time in seconds. Blue is a binary comparison (1 they match; -1 they do not match). Green is the cosine similarity. The model once again rotates into alignment at the appropriate time-step (1s). 


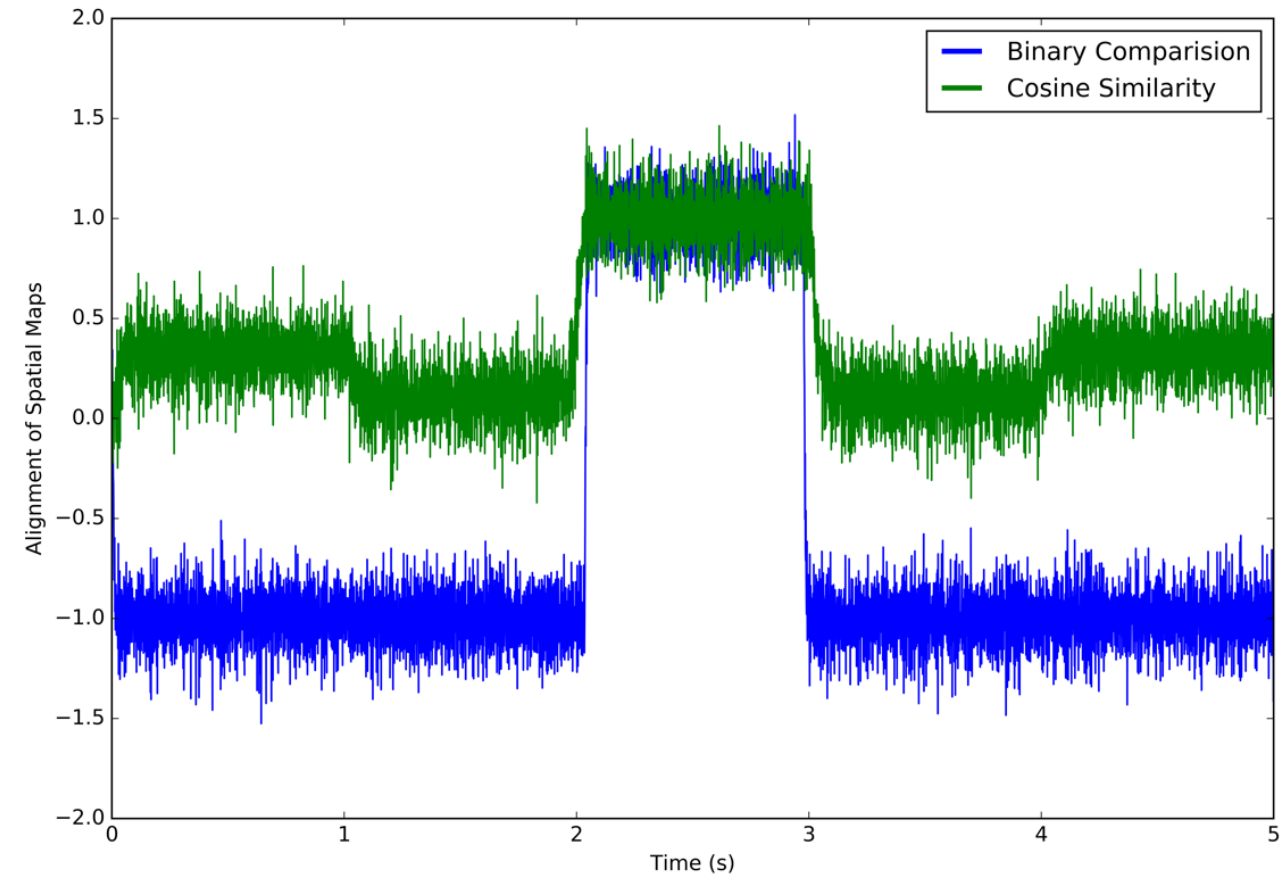

Figure 19: Simulation 12: Output Ensemble: 3 units of separation

$\mathrm{Y}$-axis is the alignment of $\mathbf{M}_{R}$ with $\mathbf{M}_{2}$. X-axis is time in seconds. Blue is a binary comparison (1 they match; -1 they do not match). Green is the cosine similarity. The model once again rotates into alignment at the appropriate time-step (2s). 


\subsection{Results Summary}

Overall, the model can successfully perform both two-dimensional and threedimensional rotation by bringing $\mathbf{M}_{1}$ into alignment with $\mathbf{M}_{2}$ (evaluation criteria i), and can do so such that RTs scale linearly with the units of angular separation (evaluation criteria ii). This is what we would expect a successful model to do given behavioural findings (see Section 3.1). That said, computing limitations means that there is a tradeoff between the biologically accuracy of neurons and the biological accuracy of spatial maps, with representational drift occurring in both sets of simulations. These pitfalls can be greatly reduced by increasing the number of neurons in the model, thus allowing for more accurate encodings of the state-space. As such, I contend these pitfalls do not invalidate the my results, and that the model lays a foundation for future research. Finally, I limited rotations to a single direction along a single axis; however, rotations can be performed in any direction along any axis by altering $\Psi$ and $M M$ as needed. 


\section{Chapter: Discussion}

There is certainly a debate to be had over the nature of mental imagery, and even though this thesis has only scratched the issue's surface, it is apparent the matter is deeply nuanced. Within this nuance, and pertinent to this thesis, is whether or not a matrix structure constitutes a depiction. As discussed in Sections 2.6 and 2.7, there is an argument to be made that matrices are descriptive as they not only exist independent of any coordinate system, but also enable easy movement between any pair of locations. On the other hand, any data structure must be understood in terms of a processing system, which may end up bestowing depictive properties upon matrices. In this thesis, I have drawn on concepts from both sides of the debate: the model is capable of shifting matrix data in a way that does not engender a continuous rotation (it can easily move data to any other location in the matrix), but by treating the all the data in the matrix as a connected unit that must move in unison, even if a non-continuous rotation were produced, the matrix is still being processed in such a way that it has depictive properties. Of course, this necessitates a spatial/indexing system, which in this thesis is thought to come from the matrix residing within an egocentric frame of reference. We saw evidence in Chapter 4 that the brain may process visual inputs in terms of a coordinate system (not just coordinate-free propositions), so the idea that some sort of coordinate system plays a role in frames of reference is not baseless. However, perception is separate from imagery, and although there is overlap between the two (see Section 2.4), the matter is far from settled.

More importantly, there is the larger question of whether spatial maps (and mental images more generally) are immutable. We can absolutely imagine one thing - an 
elephant, for example - then alter its properties - replace the elephant's trunk with a parrot's beak - but is this alteration done to the original mental image, or is a new mental image containing this alteration formed? Throughout this thesis I have continually made a distinction between $\mathbf{M}_{R}$ and $\mathbf{M}_{1}$. This was not born of a notational convenience; this was done because I contend that $\mathbf{M}_{R}$ is a not simply $\mathbf{M}_{1}$ that has been altered "in-place," but instead exists as a distinct entity.

To this end, there is a noted division between spatial representation and spatial transformation, with evidence that spatial transformations occur when a spatial map is passed to different brain regions (see Thompson et al., 2009). However, the role all of these regions play in spatial imagery cannot be fully understood from the static nature of neural imaging, thus we are forced to speculate, with my hypothesis being that spatial transformation networks do not act on representation networks, but in response to representation networks. That is, location and motor information reside in a representation network, which connects to a transformation network that interprets said information so-as-to produce a new representation with the updated orientation. If we go the other way and assume transformation networks act on representation networks (Figure 20), then $\Psi$ would have to be static, as opposed to dynamic. This approach would result in a single rule that gets applied to each new orientation of the spatial map (e.g., "take the current orientation and move it one unit"), as opposed to a continuously evolving instruction that facilitates movement from the static, canonical orientation, to every other orientation along an axis of rotation. 


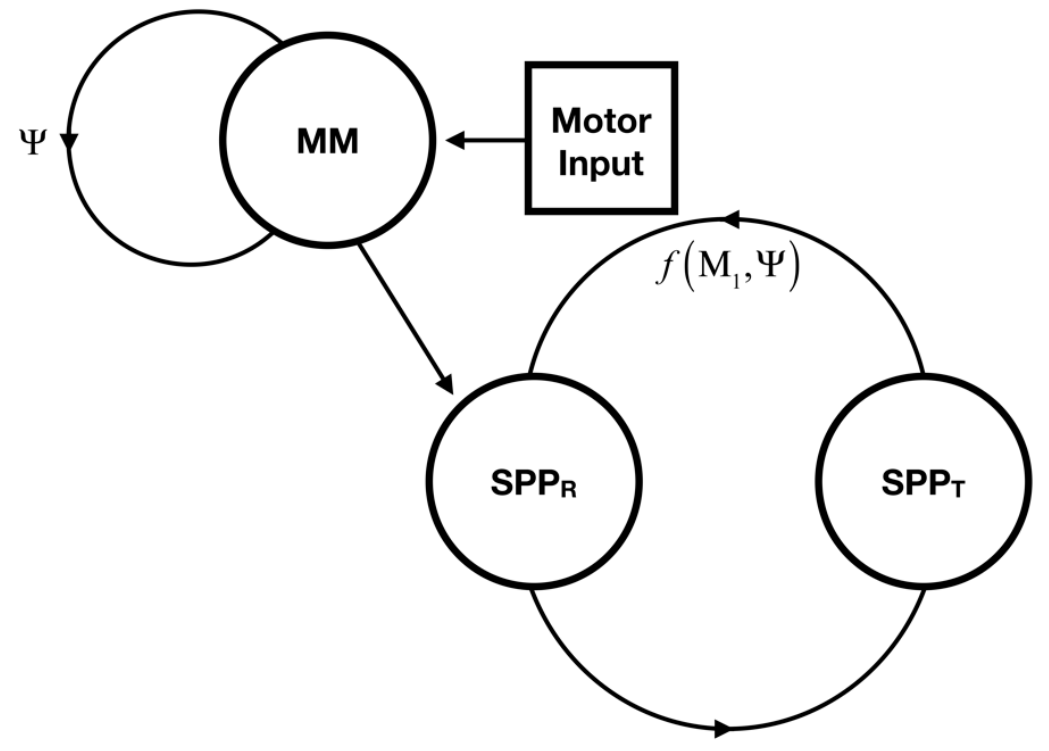

Figure 20: Transformation Network Acting on Representation Network

Here, $\mathbf{S P P} \mathbf{P}_{\mathbf{T}}$ receives $\Psi$ and the current orientation of $\mathbf{M}_{\mathbf{1}}$, then computes the rotation, with the updated orientation then being formed in $\mathbf{S P} \mathbf{P}_{\mathbf{R}}$. Crucially, this requires $\Psi$ be static as each loop results in a new orientation being sent to $\mathbf{S P P}_{\mathbf{T}}$. 
This is a nuanced point: the former requires that rotation involve constant and exact knowledge of the spatial map's orientation, whereas the latter does not. Given that motor regions have been linked to mental rotation (see Section 3.2), and that motor regions can necessarily facilitate physical object rotation without having constant and exact knowledge of the object's orientation (I do not need constant and exact knowledge of a golf ball's orientation in order to physically rotate it!), it seems a static rule is unlikely as that would imply motor regions facilitate rotation in different ways for mental and physical rotation, instead of having a single method that operates across both. In fact, there is evidence that mental rotation and low-level visual processing do not interact (Jolicoeur \& Cavangah, 1992), which is the opposite of what one would expect if constant and exact knowledge of orientation was required for mental rotation. (There is evidence that watching video of an object rotating, then repeating that rotation mentally, results in a mental rotation strategy that does not involve motor regions (Kosslyn et al., 2001), which implies that multiple strategies exist; however, my point of contention is in having motor regions operate in two different ways depending on physical/mental rotation).

All of this is not to say orientation is irrelevant to mental rotation (the canonical orientation is certainly relevant, as is the target orientation), I simply contend that continuous and exact knowledge of orientation is not needed, as would be required if transformation networks acted on representation networks. Instead, there is evidence that simulated hand movements are implicated in mental rotation (e.g., Guy et al., 2002; Wohlschläger \& Wohlschläger, 1998; Wraga et al., 2002), which suggests to me that the MM functions to perform these simulated movements (which will necessarily be 
continuous, though they may not be conscious) so-as-to define movement along the axis of rotation. $f\left(\mathbf{M}_{1}, \Psi\right)$ then functions to take this movement and determines what $\mathbf{M}_{1}$ would look like if it were physically rotated, building iterations of a new spatial map, $\mathbf{M}_{R}$, in the process. This is made possible thanks to the properties of matrices, namely that one can easily move to-and-from any location within them (see Section 2.6). A potential counter argument to this formulation would be that, when transformation networks act on representation networks, they only require an estimate of the current orientation; however, this still requires constant knowledge of the estimate, as well as a static rotation instruction. This counter-argument is certainly plausible (albeit unlikely), thus the question of how these two networks relate to one another largely comes down to how motor regions operate during imagery.

There is EEG and time-resolved fMRI evidence that motor regions are active for the duration of mental rotation (Pegna et al., 1997; Richter et al., 2000), which suggests to me a dynamic rotation instruction, but could also be weakly explained by the constant application of a static rule. However, if mental rotation was to depend on a static rule, then simultaneous acts of mental and physical rotation should not interact. This is not the case as simultaneously performing mental and physical rotation along the same axis leads to increased RTs (Wohlschläger \& Wohlschläger, 1998), which suggests mental rotation involves a dynamic instruction regarding movement along an axis, with the increased RTs resulting from attempts to simultaneously engage with two movement instances (physical hand versus simulated hand) across the same axis. On the other hand (pun very much intended), one could argue that the increased RTs are a byproduct of attempting to simultaneously engaged with two orientations (the mental image and the physical object); 
however, I have already elucidated a variety of reasons to be uncomfortable with this notion, to say nothing of how the paradigm used in Wohlschläger and Wohlschläger (1998) prevented participants from visually attending to both the physical object (a uniform, ball-shaped knob) and the imagery stimuli (Shepard and Metzler cube assemblies). A supplemental argument could be made that the knob's orientation is/was generated from tactile information, though my model is further, albeit tangentially, supported by: (i) single-unit recordings of monkeys' primary motor cortex that show hand rotational velocity is represented far more in neuron firing rates than hand orientation during hand rotation and translation tasks (Wang et al., 2010), which suggests that movement is given precedence over orientation; and, (ii) simulations demonstrating how grid cells in rats are able to use velocity and heading to generate a good estimate of position in two-dimensional space (Burak \& Fiete, 2009), which lends supplemental support to my hypothesis that the computations involved in the formation of rotated orientations are largely driven by movement, not orientation.

Following from all this, the linear RTs produced by my model are a function of MM, not the underlying form of the mental image. A reasonably strong case can be made that mental rotation is not cognitively penetrable and is constrained by our underlying biology (see Section 3.1). But other imagery tasks are cognitively penetrable (see Section 2.3), which suggests that it may not be the underling form of mental images that impose these constraints. Kosslyn, Thompson, and Ganis (2006) have proposed a solution to this dichotomy that preserves the constraining nature of imagery's underlying form (see Section 2.6); however, the simpler solution is to offload constraints to processes that operate on mental images, which can help reconcile some of mental 
imagery's disparate behavioural findings (see Riley \& Davies, Forthcoming). In the case of mental rotation, this process is found in the MM, which, as I theorized above, produces simulated hand movements that are necessarily continuous, thus leading to continuous rotations (see next paragraph) and linear RTs. Moreover, this constraint offloading has the interesting consequence of implying that individual differences in imagery are greatly influence by individual differences in the processes that operate on mental images: There are clear sex difference in mental rotation whereby males perform significantly better than females (Maeda \& Yukiko, 2013), which makes sense given the literature on sex differences in both motor performance and visual-motor abilities (Thomas \& French, 1985; Vogel, 1990); however, the matter remains largely unexplored.

Further, all of this does evoke the question of whether repeatedly mapping the canonical orientation to orientations at various positions along an axis constitutes a continuous rotation, or a series of disjointed leaps. I think it is fair to say my model computes mental rotation as a series of disjointed leaps, but the computational aspect is very different from, and need not be available to, experience of the phenomenon. Indeed, a person may have no knowledge of how their brain computes mental rotation, but that does not preclude them from experiencing the output of those computations. Thus, even though $f\left(\mathbf{M}_{1}, \Psi\right)$ involves disjointed leaps, the sequence of representations formed in $\mathrm{SPP}_{\mathrm{T}}$ are that of a continuous rotation, which is what matters as it is $\mathrm{SPP}_{\mathrm{T}}$ that connects downstream for additional processing.

That being said, by using a direct connection between $\mathrm{SPP}_{\mathrm{R}}$ and $\mathrm{SPP}_{\mathrm{T}}$ that computes $f\left(\mathbf{M}_{1}, \Psi\right)$ at every timestep, the model is implying that the computations underpinning mental rotation occur at the dendritic level. This notion is not baseless as 
dendritic computation has been observed in insects and mammals (e.g., Agmon-Snir, Carr \& Rinzel, 1998; London \& Häusser, 2005), and have not only been linked to highlevel cognitive functions such as learning (Nolan et al., 2004), but also low-level functions such as computing motion direction (Vlasits et al., 2016). Of course, whether dendritic computation occurs in humans, and during mental rotation more specifically, remains to be seen, and is an interesting avenue for future research.

At this point, I feel it is important to state that I am not the first to computationally model mental rotation in a biologically plausible way. Of particular note is the work of McKinstry et al., (2016), who used a network of spiking neurons to perform the Shepard and Metzler (1971) paradigm with a humanoid robot. However, despite the biological accuracy of the underlying neurons and the use of three-dimensional stimuli, the act of mental rotation was achieved by observing the object rotate, then learning the pattern of neural activation required to perform mental rotations (McKinstry et al., 2016). Although this parallels a known strategy (see Kosslyn et al., 2001), it is not the dominant one. In a related vein Seepanomwan et al., (2013) and Seepanomwan (2017) also took an embodied cognition approach, though their stimuli were two-dimensional. A humanoid robot (simulated in Seepanomwan et al., 2013) performed the Shepard and Metzler (1971) paradigm using a biologically informed architecture called TRoPICALS (Caligiore et al., 2010); however, the task was performed in a way that required (i) constant monitoring of the object's orientation, (ii) model training (Hebbian leaning), and (iii) the pre-activation of all possible orientations (with the model sequentially walking through each orientation until a match was found), thus suggesting a better approach might exist. Moreover, none of these models give much attention to the cognitive 
underpinnings of mental rotation (e.g., how there are different strategies depending on stimulus complexity; how frames of reference and familiar orientations factor in; how spatial maps are constructed and represented; etc.). There is certainly value in the biological constraints of their models, but biology and cognition are two parts of the same equation, and attending to one but not the other does not facilitate a sufficently in-depth and accurate understanding of the phenomenon. In fact, this is a key reason why Nengo is such a powerful tool: it allows one to model cognition, not just replicate overt behaviour such as button presses, within the confines of biological constraints.

Overall, I have presented a model that is capable of mentally rotating both twoand-three-dimensional objects. The model resides within a biologically plausible framework (Nengo), and provides a reasonable account of how the known biology and psychology may fit together. Moreover, the model coincides with the dominate theory of mental imagery (see Kosslyn, Thompson, \& Ganis, 2006), with the only modification being a more fine-grain outline of how the SPP relates to the visual buffer: specifically, any task involving the transformation of a spatial map will tap into $\mathrm{SPP}_{\mathrm{T}}$, with any task involving a static spatial map tapping into $\mathrm{SPP}_{\mathrm{R}}$. Moreover, the model can be extended to account for multi-object scenes by invoking $\mathbf{M}^{+}$, as well as other dominate rotation strategies, such as the piecewise rotation of complex and unfamiliar stimuli. However, there is currently a trade-off between the accuracy of neurons and the accuracy of spatial maps. It takes a large number of neurons to effectively encode state-spaces as large as the ones used in this thesis (36 and 72 dimensions), which requires significant computing power. Nevertheless, the fact such a large model is capable of performing a complex cognitive task on consumer hardware speaks volumes to power of Nengo. In sum, I 
contend this thesis presents a small but important step towards a deeper understanding of both mental rotation and mental imagery. 


\section{References}

Agmon-Snir, H., Carr, C. E., \& Rinzel, J. (1998). The role of dendrites in auditory coincidence detection. Nature, 393(6682), 268.

Anderson, J. R. (1978). Arguments concerning representations for mental imagery. Psychological Review, 85(4), 249.

Bekolay, T., Bergstra, J., Hunsberger, E., DeWolf, T., Stewart, T. C., Rasmussen, D., ... Eliasmith, C. (2014). Nengo: a Python tool for building large-scale functional brain models. Frontiers in Neuroinformatics, 7, 48.

Bethell-Fox, C. E., \& Shepard, R. N. (1988). Mental rotation: Effects of stimulus complexity and familiarity. Journal of Experimental Psychology: Human Perception and Performance, 14(1), 12.

Borst, G., Kievit, R. a., Thompson, W. L., \& Kosslyn, S. M. (2011). Mental rotation is not easily cognitively penetrable. Journal of Cognitive Psychology, 23(1), 60-75.

Burak, Y., \& Fiete, I. R. (2009). Accurate path integration in continuous attractor network models of grid cells. PLoS Computational Biology, 5(2), e1000291.

Caligiore, D., Borghi, A. M., Parisi, D., \& Baldassarre, G. (2010). TRoPICALS: A computational embodied neuroscience model of compatibility effects. Psychological Review, 117(4), 1188.

Charniak, E. (1991). Bayesian networks without tears. AI Magazine, 12(4), 50.

Cooper, L. A. (1976). Demonstration of a mental analog of an external rotation. Perception \& Psychophysics, 19(4), 296-302.

Davis, E. (2011). How does a box work? A study in the qualitative dynamics of solid objects. Artificial Intelligence, 175(1), 299-345.

Davis, E., Marcus, G., \& Frazier-Logue, N. (2017). Commonsense reasoning about containers using radically incomplete information. Artificial Intelligence, 248, 4684.

Denis, M., \& Cocude, M. (1989). Scanning visual images generated from verbal descriptions. European Journal of Cognitive Psychology, 1(4), 293-307.

DeWolf, T., \& Eliasmith, C. (2011). The neural optimal control hierarchy for motor control. Journal of Neural Engineering, 8(6), 65009. 
Ekstrom, A. D., Arnold, A. E. G. F., \& Iaria, G. (2014). A critical review of the allocentric spatial representation and its neural underpinnings: toward a networkbased perspective. Frontiers in Human Neuroscience, 8, 803.

Eliasmith, C., Stewart, T. C., Choo, X., Bekolay, T., DeWolf, T., Tang, C., ... Rasmussen, D. (2012). A Large-Scale Model of the Functioning Brain. Science, $338(6111), 1202-1205$.

Eliasmith, C. (2013). How to build a brain: A neural architecture for biological cognition. Oxford University Press.

Farah, M. J., Hammond, K. M., Levine, D. N., \& Calvanio, R. (1988). Visual and spatial mental imagery: Dissociable systems of representation. Cognitive Psychology, 20(4), 439-462.

Filimon, F. (2015). Are all spatial reference frames egocentric? Reinterpreting evidence for allocentric, object-centered, or world-centered reference frames. Frontiers in Human Neuroscience, 9, 648.

Fodor, J. A. (1975). The language of thought (Vol. 5). Harvard University Press.

Fox, D. (2007). Free choice and the theory of scalar implicatures. In Presupposition and implicature in compositional semantics (pp. 71-120). Springer.

Galton, F. (1880). Statistics of mental imagery. Mind, 5(19), 301-318.

Goldston, D. B., Hinrichs, J. V, \& Richman, C. L. (1985). Subjects' expectations, individual variability, and the scanning of mental images. Memory \& Cognition, 13(4), 365-370.

Goodman, D. F. M., \& Brette, R. (2009). The brian simulator. Frontiers in Neuroscience, $3,26$.

Grant, P., \& Rubin, E. (1980). Ontogeny of the retina and optic nerve in Xenopus laevis. II. Ontogeny of the optic fiber pattern in the retina. Journal of Comparative Neurology, 189(4), 671-698.

Grootswagers, T., Kooiker, B., \& Sloff, L. (2012). Applications of Bayesian Network Models in Cognitive Science.

Hegarty, M., \& Waller, D. (2004). A dissociation between mental rotation and perspective-taking spatial abilities. Intelligence, 32(2), 175-191.

Hollup, S. A., Molden, S., Donnett, J. G., Moser, M.-B., \& Moser, E. I. (2001). Accumulation of hippocampal place fields at the goal location in an annular watermaze task. Journal of Neuroscience, 21(5), 1635-1644. 
Jolicoeur, P., \& Cavanagh, P. (1992). Mental rotation, physical rotation, and surface media. Journal of Experimental Psychology: Human Perception and Performance, $18(2), 371$.

Just, M. A., \& Carpenter, P. A. (1985). Cognitive coordinate systems: accounts of mental rotation and individual differences in spatial ability. Psychological Review, 92(2), 137.

Kamp, H., \& Reyle, U. (2013). From discourse to logic: Introduction to modeltheoretic semantics of natural language, formal logic and discourse representation theory (Vol. 42). Springer Science \& Business Media.

Knauff, M., Kassubek, J., Mulack, T., \& Greenlee, M. W. (2000). Cortical activation evoked by visual mental imagery as measured by fMRI. Neuroreport, 11(18), $3957-$ 3962.

Kosslyn, S. M., \& Pomerantz, J. R. (1977). Imagery, propositions, and the form of internal representations. Cognitive Psychology, 9(1), 52-76.

Kosslyn, S. M., Ball, T. M., \& Reiser, B. J. (1978). Visual images preserve metric spatial information: evidence from studies of image scanning. Journal of Experimental Psychology: Human Perception and Performance, 4(1), 47.

Kosslyn, S. M., Thompson, W. L., Klm, I. J., \& Alpert, N. M. (1995). Topographical representations of mental images in primary visual cortex. Nature, 378(6556), 496.

Kosslyn, S. M. (1996). Image and brain: The resolution of the imagery debate. MIT press.

Kosslyn, S. M., Thompson, W. L., Wraga, M., \& Alpert, N. M. (2001). Imagining rotation by endogenous versus exogenous forces: Distinct neural mechanisms. NeuroReport, 12(11), 2519-2525.

Kosslyn, S. M., \& Thompson, W. L. (2003). When is early visual cortex activated during visual mental imagery? Psychological Bulletin, 129(5), 723.

Kosslyn, S. M., Thompson, W. L., \& Ganis, G. (2006). The case for mental imagery. Oxford University Press.

London, M., \& Häusser, M. (2005). Dendritic computation. Annu. Rev. Neurosci., 28, 503-532.

Longuet-Higgins, H. C., \& Prazdny, K. (1980). The interpretation of a moving retinal image. In Proc. R. Soc. Lond. B (Vol. 208, pp. 385-397). The Royal Society. 
MacDonald, N. (1977). A polar co-ordinate system for positional information in the vertebrate neural retina. Journal of Theoretical Biology, 69(1), 153-165.

Maeda, Y., \& Yoon, S. Y. (2013). A meta-analysis on gender differences in mental rotation ability measured by the Purdue spatial visualization tests: Visualization of rotations (PSVT: R). Educational Psychology Review, 25(1), 69-94.

Marmor, G. S., \& Zaback, L. a. (1976). Mental rotation by the blind: does mental rotation depend on visual imagery? Journal of Experimental Psychology. Human Perception and Performance, 2(4), 515-521.

McKinstry, J. L., Fleischer, J. G., Chen, Y., Gall, W. E., \& Edelman, G. M. (2016). Imagery may arise from associations formed through sensory experience: a network of spiking neurons controlling a robot learns visual sequences in order to perform a mental rotation task. PloS One, 11(9), e 0162155.

McNaughton, B. L., Battaglia, F. P., Jensen, O., Moser, E. I., \& Moser, M.-B. (2006). Path integration and the neural basis of the'cognitive map'. Nature Reviews Neuroscience, 7(8), 663.

Mellet, E., Tzourio, N., Crivello, F., Joliot, M., Denis, M., \& Mazoyer, B. (1996). Functional anatomy of spatial mental imagery generated from verbal instructions. Journal of Neuroscience, 16(20), 6504-6512.

Moser, E. I., Kropff, E., \& Moser, M.-B. (2008). Place cells, grid cells, and the brain's spatial representation system. Annual Review of Neuroscience, 31.

Nengo Source Code: https://www.nengo.ai/nengo/_modules/index.html

Nolan, M. F., Malleret, G., Dudman, J. T., Buhl, D. L., Santoro, B., Gibbs, E., ... Kandel, E. R. (2004). A behavioral role for dendritic integration: HCN1 channels constrain spatial memory and plasticity at inputs to distal dendrites of CA1 pyramidal neurons. Cell, 119(5), 719-732.

Pavlides, C., \& Winson, J. (1989). Influences of hippocampal place cell firing in the awake state on the activity of these cells during subsequent sleep episodes. Journal of Neuroscience, 9(8), 2907-2918.

Pegna, A. J., Khateb, A., Spinelli, L., Seeck, M., Landis, T., \& Michel, C. M. (1997). Unraveling the cerebral dynamics of mental imagery. Human Brain Mapping, 5(6), 410-421.

Peters, S., \& Shrobe, H. E. (2003). Using semantic networks for knowledge representation in an intelligent environment. In Pervasive Computing and Communications, 2003.(PerCom 2003). Proceedings of the First IEEE International Conference on (pp. 323-329). IEEE. 
Petit, L. S., Pegna, A. J., Mayer, E., \& Hauert, C.-A. (2003). Representation of anatomical constraints in motor imagery: mental rotation of a body segment. Brain and Cognition, 51(1), 95-101.

Pylyshyn, Z. W. (1973a). What the mind's eye tells the mind's brain: A critique of mental imagery. Psychological Bulletin, 80(1), 1.

Pylyshyn, Z. W. (1973b). The role of competence theories in cognitive psychology. Journal of Psycholinguistic Research, 2(1), 21-50.

Pylyshyn, Z. W. (1981). The imagery debate: Analogue media versus tacit knowledge. Psychological Review, 88(1), 16.

Pylyshyn, Z. W. (2002). Mental Imagery: In search of a theory. Behavioral and Brain Science, 25, 157-238.

Pylyshyn, Z. W. (2003). Seeing and visualizing: It's not what you think. MIT press.

Rasmussen, D., \& Eliasmith, C. (2014). A spiking neural model applied to the study of human performance and cognitive decline on Raven's Advanced Progressive Matrices. Intelligence, 42, 53-82.

Richter, W., Somorjai, R., Summers, R., Jarmasz, M., Menon, R. S., Gati, J. S., ... Kim, S.-G. (2000). Motor area activity during mental rotation studied by time-resolved single-trial fMRI. Journal of Cognitive Neuroscience, 12(2), 310-320.

Röder, B., \& Rösler, F. (1998). Visual input does not facilitate the scanning of spatial images. Journal of Mental Imagery.

Rosenbloom, P. S. (2011). Mental Imagery in a Graphical Cognitive Architecture. In $B I C A$ (pp. 314-323).

Schultheis, H., \& Barkowsky, T. (2011). Casimir: an architecture for mental spatial knowledge processing. Topics in Cognitive Science, 3(4), 778-795.

Schwartz, E. L. (1984). Anatomical and physiological correlates of visual computation from striate to infero-temporal cortex. IEEE Transactions on Systems, Man, and Cybernetics, (2), 257-271.

Seepanomwan, K., Caligiore, D., Baldassarre, G., \& Cangelosi, A. (2013). Modelling mental rotation in cognitive robots. Adaptive Behavior, 21(4), 299-312.

Seepanomwan, K. (2017). Generalization of a mental rotation skill in humanoid robots. In Computer Science and Software Engineering (JCSSE), 2017 14th International Joint Conference on (pp. 1-6). IEEE. 
Shepard, R. N., \& Metzler, J. (1971). Mental Rotation of Three-Dimensional Objects. Science, 171(3972), 701-703.

Shortliffe, E. (2012). Computer-based medical consultations: MYCIN (Vol. 2). Elsevier.

Singh, R., \& Eliasmith, C. (2006). Higher-dimensional neurons explain the tuning and dynamics of working memory cells. Journal of Neuroscience, 26(14), 3667-3678.

Slotnick, S. D., Thompson, W. L., \& Kosslyn, S. M. (2005). Visual mental imagery induces retinotopically organized activation of early visual areas. Cerebral Cortex, 15(10), 1570-1583.

Stenning, K., \& Oberlander, J. (1995). A cognitive theory of graphical and linguistic reasoning: Logic and implementation. Cognitive Science, 19(1), 97-140.

Tarr, M. J., \& Pinker, S. (1989). Mental rotation and orientation-dependence in shape recognition. Cognitive Psychology, 21(2), 233-282.

Tarr, M. J., \& Pinker, S. (1990). When does human object recognition use a viewercentered reference frame? Psychological Science, 1(4), 253-256.

Tarr, M. J. (1995). Rotating objects to recognize them: A case study on the role of viewpoint dependency in the recognition of three-dimensional objects. Psychonomic Bulletin \& Review, 2(1), 55-82.

Thomas, J. R., \& French, K. E. (1985). Gender differences across age in motor performance: A meta-analysis. Psychological Bulletin, 98(2), 260.

Thompson, W. L., Slotnick, S. D., Burrage, M. S., \& Kosslyn, S. M. (2009). Two forms of spatial imagery: Neuroimaging evidence. Psychological Science, 20(10), 12451253.

Tistarelli, M., \& Sandini, G. (1993). On the advantages of polar and log-polar mapping for direct estimation of time-to-impact from optical flow. IEEE Transactions on Pattern Analysis and Machine Intelligence, 15(4), 401-410.

Tootell, R. B., Silverman, M. S., Switkes, E., \& De Valois, R. L. (1982). Deoxyglucose analysis of retinotopic organization in primate striate cortex. Science, 218(4575), 902-904.

Tootell, R. B. H., Hadjikhani, N. K., Vanduffel, W., Liu, A. K., Mendola, J. D., Sereno, M. I., \& Dale, A. M. (1998). Functional analysis of primary visual cortex (V1) in humans. Proceedings of the National Academy of Sciences, 95(3), 811-817.

Ulanovsky, N., \& Moss, C. F. (2011). Dynamics of hippocampal spatial representation in echolocating bats. Hippocampus, 21(2), 150-161. 
Van Essen, D. C., Lewis, J. W., Drury, H. A., Hadjikhani, N., Tootell, R. B. H., Bakircioglu, M., \& Miller, M. I. (2001). Mapping visual cortex in monkeys and humans using surface-based atlases. Vision Research, 41(10-11), 1359-1378.

Vingerhoets, G., De Lange, F. P., Vandemaele, P., Deblaere, K., \& Achten, E. (2002). Motor imagery in mental rotation: an fMRI study. Neuroimage, 17(3), 1623-1633.

Vlasits, A. L., Morrie, R. D., Tran-Van-Minh, A., Bleckert, A., Gainer, C. F., DiGregorio, D. A., \& Feller, M. B. (2016). A role for synaptic input distribution in a dendritic computation of motion direction in the retina. Neuron, 89(6), 1317-1330.

Vogel, S. A. (1990). Gender differences in intelligence, language, visual-motor abilities, and academic achievement in students with learning disabilities: A review of the literature. Journal of Learning Disabilities, 23(1), 44-52.

Wang, W., Chan, S. S., Heldman, D. A., \& Moran, D. W. (2010). Motor cortical representation of hand translation and rotation during reaching. Journal of Neuroscience, 30(3), 958-962.

Wohlschläger, A., \& Wohlschläger, A. (1998). Mental and manual rotation. Journal of Experimental Psychology: Human Perception and Performance, 24(2), 397.

Wraga, M., Thompson, W. L., Alpert, N. M., \& Kosslyn, S. M. (2003). Implicit transfer of motor strategies in mental rotation. Brain and Cognition, 52(2), 135-143.

Zacks, J. M. (2008). Neuroimaging studies of mental rotation: a meta-analysis and review. Journal of Cognitive Neuroscience, 20(1), 1-19.

Zetzsche, C., Krieger, G., \& Wegmann, B. (1999). The atoms of vision: Cartesian or polar? JOSA A, 16(7), 1554-1565. 